\title{
Negative Interspike Interval Correlations Increase the Neuronal Capacity for Encoding Time-Dependent Stimuli
}

\author{
Maurice J. Chacron, ${ }^{1,2}$ André Longtin, ${ }^{1}$ and Leonard Maler ${ }^{2}$ \\ ${ }^{1}$ Department of Physics, University of Ottawa, Ottawa, Ontario, Canada K1N-6N5, and 2Department of Cellular and \\ Molecular Medecine, University of Ottawa, Ottawa, Ontario, Canada K1H-8M5
}

Accurate detection of sensory input is essential for the survival of a species. Weakly electric fish use amplitude modulations of their self-generated electric field to probe their environment. P-type electroreceptors convert these modulations into trains of action potentials. Cumulative relative refractoriness in these afferents leads to negatively correlated successive interspike intervals (ISIs). We use simple and accurate models of P-unit firing to show that these refractory effects lead to a substantial increase in the animal's ability to detect sensory stimuli. This assessment is based on two approaches, signal detection theory and information theory. The former is appropriate for low-frequency stimuli, and the latter for high-frequency stimuli. For low frequencies, we find that signal detection is dependent on differences in mean firing rate and is optimal for a counting time at which spike train variability is minimal. Furthermore, we demonstrate that this minimum arises from the presence of negative ISI correlations at short lags and of positive ISI correlations that extend out to long lags. Although ISI correlations might be expected to reduce information transfer, in fact we find that they improve information transmission about timevarying stimuli. This is attributable to the differential effect that these correlations have on the noise and baseline entropies. Furthermore, the gain in information transmission rate attributable to correlations exhibits a resonance as a function of stimulus bandwidth; the maximum occurs when the inverse of the cutoff frequency of the stimulus is of the order of the decay time constant of refractory effects. Finally, we show that the loss of potential information caused by a decrease in spike-timing resolution is smaller for low stimulus cutoff frequencies than for high ones. This suggests that a rate code is used for the encoding of low-frequency stimuli, whereas spike timing is important for the encoding of high-frequency stimuli.

Key words: electrosensory afferent; electrolocation; interspike intervals; spike train variability; weak signal detection; correlations; information theory; resonance
Detecting external stimuli is essential for an animal to survive in its environment. A variety of methods have been used to quantify information transmission in sensory systems, such as information theory (Borst and Theunissen, 1999; Buračas and Albright, 1999), signal detection theory (Green and Swets, 1966; Gabbiani and Koch, 1998), and stimulus reconstruction (Rieke et al., 1997; Gabbiani and Koch, 1998). Information theory makes no assumptions on the nature of the neural code and uses mutual information to quantify information transfer in sensory systems. The mutual information $I(X, Y)$ between two random variables $X$ and $Y$ is equal to $H(X)-H(X \mid Y)$, where $H(X)$ is the entropy of $X$, and $H(X \mid Y)$ is the conditional entropy of $X$ given $Y$. Because entropy increases with variability, it is clear that $I(X, Y)$ will increase if the variability of $X$ is high and the variability of $X$ given $Y$ is low. In neural systems, we can identify a time-varying stimulus with $Y$ and a spike train with $X$. Thus, information theory suggests that high variability of the spike train in the absence of the stimulus, combined with low variability in the presence of the stimulus, will maximize information transmission. In particular, because corre-

Received Jan. 16, 2001; revised April 25, 2001; accepted May 1, 2001.

This research was supported by the Natural Sciences and Engineering Research Council of Canada (M.J.C. and A.L.) and Canadian Institutes of Health Research (L.M.) Canada. We thank B. Doiron, D. Bueti, and J. Bastian for useful discussions. We give special thanks to P. Lánský, J. Lewis, and A. M. Oswald for their careful reading of this manuscript as well as useful discussions. Finally, we thank two anonymous reviewers for their helpful comments.

Correspondence should be addressed to Maurice J. Chacron, Physics Department, 150 Louis-Pasteur, Ottawa, Ontario, Canada K1N-6N5. E-mail: mchacron@physics.uottawa.ca.

Copyright () 2001 Society for Neuroscience 0270-6474/01/215328-16\$15.00/0 lations are known to reduce entropy (Shannon, 1948), one might expect that the correlations in a spike train will degrade information transmission.

On the other hand, signal detection theory in the context of neural spike trains aims to determine the presence versus the absence of a stimulus that is based on differences between the number of spikes counted in an interval of length $T$. The discriminability $d$ between the two cases is given by the absolute value of the difference between the mean of these spike counts with and without stimulus, divided by the square root of the summed variances of these spike counts (see Eq. 6). It is clear that discriminability is enhanced by a low variability in the spike train, because this will result in low variability of spike count (low variance) over the counting window. In this paper, we show that negative interspike interval (ISI) correlations, i.e., the tendency for long ISIs to be followed by short ISIs (and vice versa), reduce spike count variability, whereas positive ISI correlations increase spike count variability. Together, these effects lead to an optimal spike counting time at which discriminability is maximal.

Contrasting the information theoretic and signal detection approaches points to an apparent contradiction with respect to spike train variability: information theory requires high variability, whereas we have just shown that signal detection requires low variability. However, we will show, in the context of the electrosensory system, that each approach is best suited to a particular stimulus frequency range. Also, we will show that negative ISI correlations enhance both the mutual information $I$ and the discriminant measure $d$. 
Gymnotiform weakly electric fish are particularly adept at detecting prey (Nelson and MacIver, 1999) and each other (Dulka et al., 1995; Bastian et al., 2001). They emit a sinusoidal timevarying electric field through their electric organ discharge (EOD; frequency, 600-1200 Hz). P-type electroreceptors on their skin detect amplitude modulations (AMs) of this field caused by nearby objects or conspecifics (for review, see Bastian, 1981; Zakon, 1986). These P-units fire action potentials when driven only by the EOD, i.e., without AMs. Therefore, detection of external stimuli is based presumably on a change from this baseline activity (Ratnam and Nelson, 2000). Furthermore, these P-units exhibit negative ISI correlations at low lags (Longtin and Racicot, 1997; Chacron et al., 2000; Ratnam and Nelson, 2000), resulting from cumulative relative refractoriness (Chacron et al., 2000).

For Apteronotus leptorhynchus (Brown ghost knife fish), P-unit spike train variability decreases (as measured by the Fano factor; see Materials and Methods) by as much as two orders of magnitude for counting times varying between 40 and 1000 EOD cycles (Ratnam and Nelson, 2000) before increasing again. Our computational study provides an explanation for this result and for the fact that P-units can transmit information about both low- and high-frequency stimuli. Our study uses simple and accurate biophysically plausible models for P-unit activity to generate the large data sets necessary for mutual information analysis and for establishing the role of the ISI correlations in the enhancement of stimulus coding.

\section{MATERIALS AND METHODS}

\section{Interspike interval analysis}

Let us denote by $\left\{I_{j}\right\}$ the ISI sequence with mean $\langle I\rangle$ and variance $\operatorname{VAR}(I)$. The coefficient of variation $(\mathrm{CV})$ of the interspike interval histogram (ISIH) provides a good measure of spike train variability on time scales of the order of the mean ISI. If $\left\{I_{j}\right\}$ is stationary, then the coefficient of variation is defined as:

$$
\mathrm{CV}=\frac{\sqrt{\operatorname{VAR}(I)}}{\langle I\rangle} .
$$

\section{Serial correlation coefficients}

One common measure of memory effects in a time series of events is the serial correlation coefficients (SCCs) $\rho_{j}$ defined for $\operatorname{lag} j$ by:

$$
\rho_{j}=\frac{\left\langle I_{j+k} I_{k}\right\rangle-\left\langle I_{k}\right\rangle^{2}}{\operatorname{VAR}(I)} .
$$

The SCCs are a measure of linear ISI correlations in the spike train.

\section{Spectral density function}

The spectral density function (SDF) is the discrete Fourier transform of the SCCs (Cox and Lewis, 1966) and is defined for positive frequencies $f$ as:

$$
\operatorname{SDF}(f)=\frac{1}{\pi}\left(1+2 \sum_{j=1}^{\infty} \rho_{j} \cos (2 \pi j f)\right) .
$$

This formula can be inverted using the inverse Fourier transform to yield an expression for $\rho_{j}$ (Cox and Lewis, 1966):

$$
\rho_{j}=2 \pi \int_{0}^{1 / 2} \operatorname{SDF}(f) \cos (2 \pi j f) d f .
$$

The spectral density function is always positive (Cox and Lewis, 1966). Moreover, specifying the SCC sequence allows us to uniquely determine the SDF and vice versa. The two quantities are thus completely equivalent (Cox and Lewis, 1966).

\section{Pulse number distributions and the Fano factor time curve}

The pulse number distribution (PND) (Barlow and Levick, 1969a,b; Teich and Khanna, 1985) $P(n, T)$ is defined as the probability of observing $n$ spikes during a counting time $T$ (the PND is sometimes referred to as the spike count distribution). It is calculated by first dividing the spike train into nonoverlapping time windows of length $T$ and then counting the number of action potentials in each window. A normalized histogram of these numbers then yields the PND. The Fano factor (Fano, 1947) is defined as the variance to mean ratio of the PND:

$$
F(T)=\frac{\sigma^{2}(T)}{\mu(T)},
$$

and has units of spikes. The Fano factor curve $F(T)$ gives a measure of spike train variability on all time scales $T$.

The Fano factor always approaches unity for small $T$ (Teich et al., 1997). For a Poisson process, we have $F(T)=1$ for any $T$ (Cox and Lewis, 1966). Processes with $F(T)<1$ are thus considered less variable than Poisson, whereas those with $F(T)>1$ are more variable (Gabbiani and Koch, 1998). The asymptotic value $F_{\infty}$ of the Fano factor is related to the SCCs of the ISI sequence (Cox and Lewis, 1966) according to:

$$
\lim _{T->\infty} F(T)=F_{\infty}=\mathrm{CV}^{2}\left(1+2 \sum_{j=1}^{\infty} \rho_{j}\right),
$$

where we have assumed that the series is convergent. Note that positive and negative ISI correlations increase and decrease, respectively, the asymptotic value $F_{\infty}$.

\section{Signal detection theory}

The ideal observer paradigm is based on the optimal discrimination between PNDs obtained in the presence and absence of a stimulus (Green and Swets, 1966) for examples of applications to neural systems (see Nachimas, 1972; Shofner and Dye, 1989; Gabbiani and Koch, 1998; Gabbiani and Metzner, 1999). Let $P_{0}(n, T)$ be the PND obtained without stimulus and let $P_{1}(n, T)$ be the one obtained in the presence of stimulus. Then, we define the probability of false alarm $P_{F A}$, i.e., of reporting a signal when it is not there, as (Gabbiani and Koch, 1998):

$$
P_{F A}(T)=\sum_{n \geq m} P_{0}(n, T),
$$

and the probability of correct detection $P_{D}$ as:

$$
P_{D}(T)=\sum_{n \geq m} P_{1}(n, T),
$$

where $m$ is some threshold. The overall performance of the detector is characterized by varying $m$ between zero and infinity and plotting $P_{D}$ as a function of $P_{F A}$. This curve is called the receiver operating characteristic (ROC) of the detector (Green and Swets, 1966; Gabbiani and Koch, 1998). The further this curve lies above the diagonal $P_{D}=P_{F A}$ (which corresponds to chance detection), the better the performance of the detector for a counting time $T$ (Gabbiani and Koch, 1998).

\section{Information theory}

The entropy of a discrete random variable $X$ with probability density function $P(X)$ is defined as (Shannon, 1948; Cover and Thomas, 1991):

$$
H(X)=-\sum_{X} P(X) \log _{2}(P(X)),
$$

and is measured in bits.

The variability of the neural response to an ensemble of stimuli is characterized by the total entropy $H_{\text {total }}$ (Strong et al., 1998; Buračas and Albright 1999) and is estimated from the neural response to an unrepeated Gaussian stimulus. The trial-to-trial variability of the neural response to a repeated stimulus is characterized by the noise entropy $H_{\text {noise }}$ (Strong et al., 1998; Reinagel and Reid, 2000) defined as the conditional entropy of the spike train given a stimulus (previously referred to as $H(X \mid Y)$ in the introductory remarks). Thus $H_{\text {noise }}$ represents the variability ("noise") in the spike train that cannot be accounted for by the stimulus. 
A good stimulus encoder must have a neural response that varies highly in response to different stimuli while having a very reliable response to repeated presentations of the same stimulus. In the case of neurons that are silent in the absence of sensory input, an estimate of the mutual information about the stimulus is given by (Strong et al., 1998):

$$
I_{\text {est }}=H_{\text {total }}-H_{\text {noise }} \text {. }
$$

This measure gives a lower bound for the mutual information, as the estimate for $H_{\text {total }}$ will be lower than the value obtained for all possible stimuli. For P-type electroreceptors, the absence of stimulus corresponds to the fish's EOD that is unmodulated by extrinsic signals. The resulting spike train defines the baseline activity of the P-unit. P-units display a phase-locked random skipping pattern $(\mathrm{CV}=0.44$ on average) (Ratnam and Nelson, 2000) to this EOD (Xu et al., 1996; Nelson et al., 1997; Chacron et al., 2000). A decision about whether a stimulus is present or not must thus presumably be made on the basis of a change from this baseline activity (Ratnam and Nelson, 2000). Hence, it is natural in our case to estimate mutual information $I$ as the difference between the entropy of the baseline activity and the entropy estimated from the trial-to-trial variability of the neural response to a repeated stimulus:

$$
I=H_{\text {baseline }}-H_{\text {noise }} .
$$

Note that we have $\mathrm{I}=0$ when no stimulus is present. We divide information and entropy by stimulus duration measured in EOD cycles and express the results in bits per EOD cycle. To estimate these quantities, we divide the spike train into bins of length $\Delta \tau$. If $n$ spikes occurred between $i \Delta \tau$ and $(i+1) \Delta \tau$, then the value assigned to bin $i$ is $n$. The entropy of words comprising $L$ bins is given by (Strong et al., 1998):

$$
H(L, \Delta \tau)=\sum_{w \in W(L, \Delta \tau)}-P(w) \log _{2} P(w),
$$

where $w$ is a word of length $L$ and $W(L, \Delta \tau)$ is the set of all possible words of length $L$. If the correlations have finite range, then we can expand $H(L, \Delta \tau)$ as a Taylor series in powers of $L^{-1}$ (Strong et al., 1998):

$$
H(L, \Delta \tau)=H(\Delta \tau)+\frac{C_{1}}{L}+\frac{C_{2}}{L^{2}} \cdots,
$$

where $H(\Delta \tau)$ is the entropy rate for infinite word length and $C_{1}$ and $C_{2}$ are constants. In some cases the $L^{-1}$ term is sufficient (Strong et al., 1998; Reinagel and Reid, 2000). However, this is not the case here; rather we performed quadratic fits of the $H(L, \Delta \tau)$ versus $L^{-1}$ data obtained to get the entropy rates and infer the information rate. We used our models to generate 1,000 realizations, each containing 10,000 successive EOD cycles with the same repeated stimulus (note that such amounts of data would have to be obtained from recordings lasting in excess of 2-3 hr, which is not currently feasible for electrosensory afferents). The baseline entropies were estimated in the same way, except that no stimulus was present.

\section{Modeling}

Because we are interested in understanding how correlations of P-unit ISIs affect information transfer, we use two models of P-type electroreceptors. The Nelson model (Nelson et al., 1997) accounts for first-order ISI statistics (i.e., the ISIH) and for the gain and phase response to sinusoidal AMs; however, it is memoryless in the sense that the significant ISI correlations displayed by experimental data are absent (see Fig. 1 and Chacron et al., 2000). The other model uses the filtering property of the Nelson model to extend the model proposed by Chacron et al. (2000). The latter model was shown to reproduce first- and second-order statistics of experimental data, namely the ISIH, ISI return map $\left(I_{j+1}\right.$ as a function of $I_{j}$ ), and the ISI correlations as measured by the SCCs. We start with a description of the Nelson model and then describe ours. For convenience, a list of all symbols and acronyms used in this paper is provided in appendix A, whereas appendix B summarizes all equations used for both models.

\section{The Nelson model}

It is known from experiments that various filtering mechanisms are at work inside a P-unit (Hopkins, 1976; Bastian, 1981; Wessel et al., 1996; Xu et al., 1996; Nelson et al., 1997). Nelson et al. (1997) measured the gain and phase response characteristics of P-units to sinusoidal AMs of frequencies in the range $0.1-200 \mathrm{~Hz}$, leading to the following set of differential equations:

$$
\begin{gathered}
\dot{X}_{a}=-\frac{X_{a}}{\tau_{a}}+\frac{G_{a}}{\tau_{a}} A(t) \\
\dot{X}_{b}=-\frac{X_{b}}{\tau_{b}}+\frac{G_{b}}{\tau_{b}} A(t) \\
X(t)=-X_{a}-X_{b}+\left(G_{a}+G_{b}+G_{c}\right) A(t),
\end{gathered}
$$

where the dot denotes differentiation with respect to time, $A(t)$ is the stimulus (i.e., the time-varying EOD amplitude minus its baseline value), and $X(t)$ is the filtered stimulus. The $G$ values are gains in units of spikes per second per millivolt, and the $\tau$ values are time constants in units of seconds. A baseline firing rate $r_{\text {base }}$ is added then to $X(t)$, and the sum $Z(t)$ is passed through a clipping nonlinearity to account for saturation effects (Nelson et al., 1997):

$$
r(t)= \begin{cases}0 & Z(t)<0 \\ f_{\mathrm{EOD}} & Z(t)>f_{\mathrm{EOD}} \\ Z(t) & \text { otherwise }\end{cases}
$$

The probability $p(t)$ of firing per EOD cycle is thus $r(t) / f_{\mathrm{EOD}}$, where $f_{\mathrm{EOD}}$ is the EOD frequency. At each maximum of the EOD, the P-unit has probability $p(t)$ of firing. If the unit fires, jitter is added to the spike time in the form of Gaussian white noise of zero mean and standard deviation 0.04 EOD cycles. Throughout this paper, we take $f_{\text {EOD }}=1000 \mathrm{~Hz}$, hence an EOD cycle corresponds to $1 \mathrm{msec}$. Furthermore, to reduce the coefficient of variation of the ISIH, it is possible to implement $m$ independent random subprocesses, each with an event rate equal to the spike rate $r(t)$ (Nelson et al., 1997). Each subprocess is simulated as described above, and output spikes are generated at the time of occurrence of every $m$ th subprocess event. The model gives an ISIH similar to the data but does not display the correlations seen experimentally (see Fig. 1 and Chacron et al., 2000). The model was constructed to give the correct responses to sinusoidal AMs (Nelson et al., 1997) and was used to give the firing dynamics in response to changes in transdermal potential caused by a prey (Nelson and MacIver, 1999).

\section{A modified integrate-and-fire type model}

Biophysical justification. We begin with a description of P-type electroreceptors to biophysically justify our model. A P-unit is composed of 25-40 receptor cells and a nerve fiber making synaptic contact onto at least 16 active neurotransmitter release sites per receptor cell (Bennett et al., 1989). Although it is currently impossible to record intracellularly from these cells, there is much indirect evidence that the EOD amplitude changes individual receptor potentials that govern the rate of release of neurotransmitter onto the afferent nerve. Fluctuations in this rate are thus one expected source of variability in such systems (Stein, 1965; Nelson et al., 1997). Another possible source is the conductance fluctuations of the ionic channels at the spike initiation zone in the axon.

As mentioned above, we expect relative refractoriness to be important at such high firing rates (Stein, 1965). It leads to negative SCCs for the ISIs at low lags (Geisler and Goldberg, 1966; Chacron et al., 2000). Because there is currently no biophysical characterization of the ionic conductances inside the P-unit, we can only speculate as to the possible mechanisms responsible for this experimentally observed relative refractoriness. The physiological mechanisms responsible for these correlations could be presynaptic in origin; for example, long-term depression at the synapses connecting the receptor cells to the afferent nerve (Bennett et al., 1989; Hausser and Roth, 1997) would lead to relative refractoriness. However, the recovery time constant of the neurotransmitter at typical synapses is usually in the thousands of milliseconds range (von Gernsdorff et al., 1997), which is much too long for the phenomenon at work here because serial correlations are significant only up to lag 2 (Chacron et al., 2000) (see Fig. 1). Thus, we are looking at a time scale of about 10 EOD cycles (twice the mean ISI, which is 5 EOD cycles long for the unit considered here). A likely candidate would be a postsynaptic spikeactivated potassium channel that slowly deactivates and thus summates to produce a negative adaptation current. The KV3.1 channel has the right activation and deactivation kinetics (Wang et al., 1998). Members of the KV3 family are richly expressed in the electrosensory system (A. J. Rashid, personal communication), but it remains to be shown whether similar channels are present in P-units. 
From the foregoing discussion, we feel that the best approach for studying the effects of correlations on information transfer and signal detection is to use a simple yet biophysically plausible model that reproduces the essential features of P-unit baseline and evoked discharge.

Description. First, we will give an expression for the synaptic current at the spike initiation zone in the axon and then describe the spiking mechanism. We use the simple model in Chacron et al. (2000) that has been proposed to model the baseline firing dynamics of P-type electroreceptors and extend it to get the proper responses to time-varying AMs of the EOD amplitude. We write the transdermal potential on the fish's skin as $\left(A(t)+A_{0}\right) \sin \left(2 \pi f_{\mathrm{EOD}} t\right)$, where $A_{0}$ is the constant EOD amplitude corresponding to baseline firing dynamics (this is similar to $r_{\text {base }}$ in Nelson's model) and $A(t)$ is the AM. The stimulus $A(t)$ is filtered using Equations 3-5. Because many receptors rectify a periodic input (French et al., 1972; Gabbiani, 1996), we take the total synaptic current to be:

$I_{\mathrm{syn}}=\left(\beta X(t)+\gamma A_{0}\right) \Theta\left(\beta X(t)+\gamma A_{0}\right) \sin \left(2 \pi f_{\mathrm{EOD}} t\right) \times$

$\Theta\left(\sin \left(2 \pi f_{\mathrm{EOD}} t\right)\right)\left(1+\lambda_{1}\right)+\lambda_{2}$,

where $\beta$ and $\gamma$ are constants to make units match, $\lambda_{1}$ and $\lambda_{2}$ are noise terms, and $\Theta$ is the Heaviside function $(\Theta(x)=1$ if $x \geq 0$ and $\Theta(x)=0$ if $x<0)$ to account for rectification. Thus, the deterministic component of the synaptic current is zero whenever $\sin \left(2 \pi f_{\mathrm{EOD}} t\right)$ is negative or $\beta X(t)+\gamma A_{0}$ is negative. This is to ensure that firings will always occur near the maxima of the EOD sine wave, as experimentally observed (Scheich et al., 1973). Because our model is phenomenological, we take the synaptic current to be dimensionless. Noise sources including conductance and synaptic fluctuations are modeled by two Ornstein-Uhlenbeck processes $\lambda_{1}$ and $\lambda_{2}$ given by:

$$
\begin{aligned}
& \dot{\lambda}_{1}=-\frac{\lambda_{1}}{\tau_{1}}+\sqrt{D_{1} \xi_{1}} \\
& \dot{\lambda}_{2}=-\frac{\lambda_{2}}{\tau_{2}}+\sqrt{D_{2}} \xi_{2}
\end{aligned}
$$

where $\xi_{1}$ and $\xi_{2}$ are two independent Gaussian random variables of zero mean and variance unity, $\mathrm{D}_{1}$ and $\mathrm{D}_{2}$ are constants proportional to the intensities of $\lambda_{1}$ and $\lambda_{2}$, and $\tau_{1}$ and $\tau_{2}$ are time constants. Figure $2 a$ gives a time series for $\lambda_{1}$ and $\lambda_{2}$. It can be shown (Gardiner, 1985) that $\lambda_{1}$ and $\lambda_{2}$ are stationary Gaussian random variables with zero mean and respective variances $D_{1} \tau_{1} / 2$ and $D_{2} \tau_{2} / 2$. However, unlike Gaussian white noise, $\lambda_{1}$ and $\lambda_{2}$ are correlated in time, and their correlation functions decay exponentially with respective time constants $\tau_{1}$ and $\tau_{2}$. We take $\tau_{1}$ to be much less than an EOD cycle; hence, $\lambda_{1}$ can be thought of as "fast" compared to the model dynamics (it is almost white noise in fact) (see Fig. 2a). It could thus model fluctuations that occur on time scales much faster than the EOD cycle (e.g., membrane noise caused by channel flicker low-pass filtered by the membrane capacitance) (Manwani and Koch, 1999). In this case, $D_{1}$ would be related to the strength of the membrane noise.

In contrast, we take $\tau_{2}$ to be much greater than the EOD cycle. Hence, this noise term can be thought of as "slow" compared with the model dynamics (it is almost constant for time scales much smaller than $\tau_{2}$ ) (see Fig. $2 a$ ). It thus models fluctuations on slow time scales (e.g., fluctuations in vesicular release rate; see below). This term is needed to accurately reproduce the Fano factor curve as shown in Results.

The spiking mechanism is a simple extension to the leaky integrateand-fire (LIF) model in which a spike is said to have occurred when the membrane potential $V$ reaches a constant threshold $\theta$. Immediately afterward, the voltage is reset to its resting value (usually taken to be 0 ). LIF models are memoryless in the sense that consecutive ISIs are not correlated $\left(\rho_{i}=0\right.$ for all $\left.i>0\right)$. To include refractory effects, we make $\theta$ also a dynamical variable (Geisler and Goldberg, 1966). But instead of making it random (Gestri et al., 1980; Gabbiani and Koch, 1996, 1998), we let it carry the memory by the following firing rule (Chacron et al., 2000): when voltage equals threshold, it is reset to zero as in the LIF model, whereas threshold is incremented by a constant amount $\Delta \theta$ and kept constant for the duration of the absolute refractory period $T_{r}$, after which it relaxes exponentially toward its equilibrium value $\theta_{0}$ until the next spiking time. The equations for voltage and threshold between times of occurrence of action potentials and after the absolute refractory period are thus:

$$
\begin{aligned}
& \dot{v}=-\frac{v}{\tau_{v}}+\frac{I_{\mathrm{syn}}}{\tau_{v}} \\
& \dot{\theta}=\left(\theta_{0}-\theta\right) / \tau_{\theta} .
\end{aligned}
$$

Like the synaptic current, $v$ and $\theta$ are dimensionless. A stretch of simulation showing $v$ and $\theta$ is shown in Figure $2 b$. The filter given by Equations 3-5 gives the linear transfer properties of the afferent, whereas our spiking mechanism gives the correct baseline dynamics. We thus combine the two to get the correct responses to AMs. Kreiman et al. (2000) had a similar approach to model the P-type electroreceptors of another species of weakly electric fish (Eigenmannia); they used a highpass filter to give proper AM response characteristics and fed the output to an LIF model. However, because their LIF model had a random threshold, it did not take into account the relative refractory effects that could exist in this species.

For the remainder of this paper, we refer to our model as the leaky integrate-and-fire with dynamic threshold (LIFDT) model. Note that our dynamic threshold can model the aforementioned KV3.1 channel but could also result from any current that leads to an increase in the effective distance between voltage and threshold immediately after a spike. It is thus very general and is used here to model cumulative refractory effects and consequently endow the ISI sequence with proper second-order statistics (Chacron et al., 2000) (Fig. 1). The experimentally obtained SCC at lag 1 for the P-receptor data was -0.35 (Chacron et al., 2000), whereas it is -0.385 for the model. Note that the three parameters for the spiking mechanism $\left(\theta_{0}, \tau_{v}\right.$, and $\left.\tau_{\theta}\right)$ can be adjusted to give the proper first- and second-order statistics (ISIH, ISI return map, and SCCs) for other P-units with different firing rates (data not shown). This spiking model could thus be used to model other neural systems in which negative ISI correlations at short lags have been observed (e.g., the auditory system) (Lowen and Teich, 1992). The reason explaining why the dynamic threshold gives rise to negative ISI correlations can be understood as follows: suppose an ISI shorter than $\langle I\rangle$ just occurred, then the threshold (having had less time to decay) will typically be high after the spike and will thus take a long time to decay. Consequently, the next ISI will (on average) be longer than $\langle I\rangle$. This gives rise to a negative SCC at lag 1. This is also the case when an ISI longer than $\langle I\rangle$ occurs; as a result, the threshold will now be lower, and the next ISI will be shorter than $\langle I\rangle$. However, because of the strong noise $\lambda_{1}$, negative ISI correlations at longer lags will be washed out. An extended explanation of the role of the noise in the model and why the dynamic threshold leads to negative ISI correlations in the presence of noise can be found in Chacron et al. (2001).

Also, our extended model gives the correct responses to sinusoidal AMs (see Fig. 3 and the next section).

\section{Stimulation}

Baseline firing statistics were computed for both models. Their ability to encode time-varying stimuli was tested using AMs of the EOD amplitude. For the LIFDT model, the EOD amplitude minus its baseline value was given by:

$$
A(t)=\sigma_{\text {stim }} s(t)
$$

where $s(t)$ is the stimulus and $\sigma_{\text {stim }}$ is the contrast. To calibrate the model, we first used sinusoidal AMs of different frequencies and intensities to construct the phase and gain response curves (see Fig. 3). We took the baseline transdermal potential to have a root mean squared value of $0.566 \mathrm{mV}$, which is in the physiological range (Xu et al., 1996; Nelson et al., 1997). The gain and phase curves were constructed then using the method outlined in Nelson et al. (1997). The gain for sinusoidal AMs of frequency $1 \mathrm{~Hz}$ was 1060 spikes per second per millivolt, which is in the experimentally observed range of values (Nelson et al., 1997).

The stimulus $c \sigma_{\text {stim }} s(t)$ was presented then to the Nelson model, and the constant $c$ was adjusted so that both models gave identical gains and phases over the frequency range of the sinusoidal AMs. For the purpose of quantifying the amount of information transmitted, we then took $s(t)$ to be low-pass filtered Gaussian white noise of mean 0 and variance 1 . A Butterworth fourth-order filter was used with cutoff frequency $f_{c}$ (Wessel et al., 1996). As mentioned above, this type of stimulus has been used widely in quantifying the ability of neurons to encode time-varying stimuli by means of the stimulus reconstruction technique (Gabbiani, 
1996; Gabbiani and Koch, 1996, 1998; Wessel et al., 1996; Chacron et al., 2000; Kreiman et al., 2000).

\section{RESULTS}

We first explain how negative ISI correlations (Fig. 1) lead to a decrease in spike train variability and how positive ISI correlations increase this variability. We then show how a combination of strong negative ISI correlations at short lags and weak positive ISI correlations induced by a weak correlated noise (Fig. 2) and extending out to long lags gives rise to the minimum in the Fano factor curve $F(T)$ seen experimentally by Ratnam and Nelson (2000). We consider the role of these ISI correlations for signal detection using the ideal observer paradigm by considering two models (see Materials and Methods) that have identical responses to sinusoidal AMs (Fig. 3). We then study their contribution to the ability of the receptor to encode time-varying stimuli in the form of low-pass filtered Gaussian white noise. This will be done by computing the mutual information rate as a function of stimulus contrast and cutoff frequency. Finally, we show that signal detection is best suited for low-frequency stimuli (e.g., electrolocation signals) (Nelson and MacIver, 1999) because spike train variability is low on long time scales, and that information theory is best suited for high-frequency stimuli (e.g., electrocommunication signals) (Zupanc and Maler, 1993; Bastian et al., 2001) because spike train variability is high on short time scales.

\section{Fano factor}

The Fano factor curve obtained for the LIFDT model with $D_{2}=$ 0 is plotted in Figure 4 (triangles). We see that the electroreceptor is more regular at all time scales than a Poisson process because $F(T)<1$ (Cox and Lewis, 1966). $F(T)$ decreases for $T$ in the 1-5000 EOD cycle range and has an asymptotic value of 0.00685 $(n=5$ line $)$. If we take only the SCCs to be nonzero up to lag 5 , then we get $F_{\infty}=0.00681$ from Equation 1 , which is very close to the observed asymptotic value of 0.00685 . For comparison, the Fano factor time curve obtained by random shuffle of the ISI sequence is also plotted in Figure 4 (diamonds). Because all ISI correlations have been eliminated by this operation, we now have a renewal process (Cox and Lewis, 1966) for which $F(T)$ tends toward $\mathrm{CV}^{2}$ from Equation $1\left(\mathrm{CV}^{2}\right.$ line in Fig. 4). Note that because $\mathrm{CV}^{2} \approx 0.0436<1$ in our case, hence we have $F_{\infty}<1$ even in the absence of ISI correlations. The two curves (triangles and diamonds) are almost on top of one another for short counting times ( $<10$ EOD cycles), implying that correlations do not play a significant role over this range from the Fano factor curve perspective; however, as we will see below, this is not the case from an information theoretic perspective. However, they become different for longer times; the Fano factor curve without correlations tends toward $\mathrm{CV}^{2}$, whereas the one with correlations has a lower asymptotic value. We plot the Fano factor curve that was obtained with the Nelson model in Figure 4 (squares). We see that it matches the one obtained for randomly shuffled ISI sequences from the LIFDT model. This match is not surprising, because the two models have for all practical purposes identical ISI distributions and thus identical CVs (Fig. 1). Furthermore, it demonstrates that there are no significant ISI correlations in the Nelson model. Because the SCCs are effectively negligible beyond lag 5 in the model, the Fano factor tends toward a constant for long counting times given by Equation 1; this is not what is observed experimentally.

We now plot the Fano factor curve obtained with $D_{2}=9 \times$

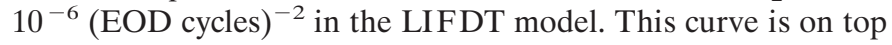
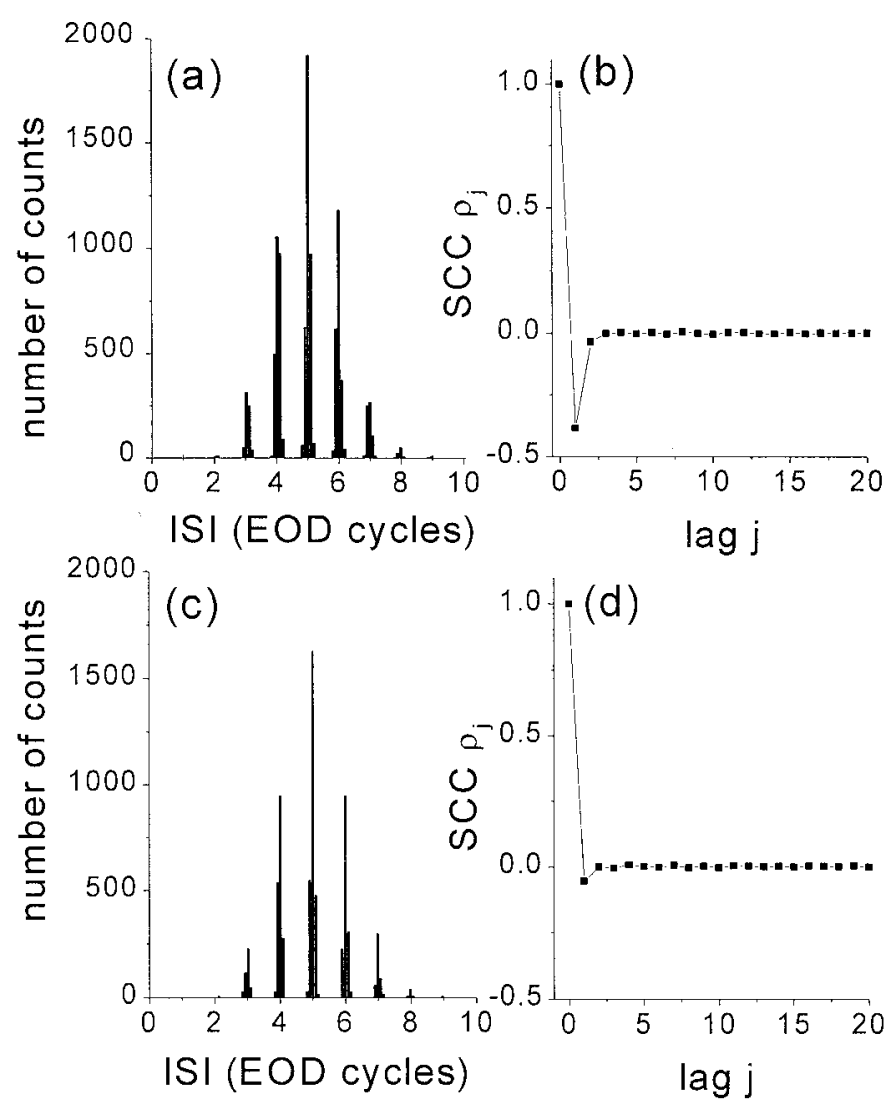

Figure 1. Comparison of the two models used in our study. a, ISIH obtained from the analysis of 10,000 consecutive ISIs from the LIFDT model $\left[\langle I\rangle=4.9912\right.$ EOD cycles, $\operatorname{VAR}(I)=1.1449 \mathrm{EOD}$ cycles $^{2}, \mathrm{CV}=$ 0.2143 ]. $b$, SCCs obtained with the model. $c$, ISIH obtained from 10,000 consecutive ISIs with Nelson's model $[\langle I\rangle=4.9982$ EOD cycles, VAR $(I)$ $=1.1003$ EOD cycles, $\mathrm{CV}=0.2098]$. $d$, SCCs obtained. Note that both models have the same distribution of ISIs but that the Nelson model does not exhibit any significant ISI correlations; the small negative SCC at lag 1 is not significant because the model has the same Fano factor curve as our model with shuffled ISIs (Fig. 4).

of the other ones for short counting times. In particular, the noise $\lambda_{2}$ is weak (Fig. 2a) and has negligible effect on the ISIH and the SCCs at low lags (see below). However, the Fano factor curve differs from the others by increasing in a power law fashion for long counting times before saturating. The behavior can be understood from a plot of the mean and variance of the PND (Fig. 5 ). The mean increases linearly with counting time; this is because the mean number of spikes that is expected in a time window of length $T$ is equal to the length of that window multiplied by the mean firing rate. However, the variance is almost constant for short counting times; hence, $F(T)$ decreases. The variance then increases at a greater rate than the mean; hence, $F(T)$ increases. Finally, the variance and mean both increase with the same rate, and the Fano factor is constant.

We now show that the increase in the Fano factor at long counting times is caused by the presence of weak positive SCCs that extend out to long lags. These positive correlations are extremely small and cannot be seen from a plot of the SCCs $\rho_{j}$ as a function of $j$. However, their presence is revealed by the increase of the spectral density function at low frequencies (Cox and Lewis, 1966) (Fig. 6). This can be seen on the following simple example in which the following form for the SCCs at lags $>0$ is assumed: 
(a)

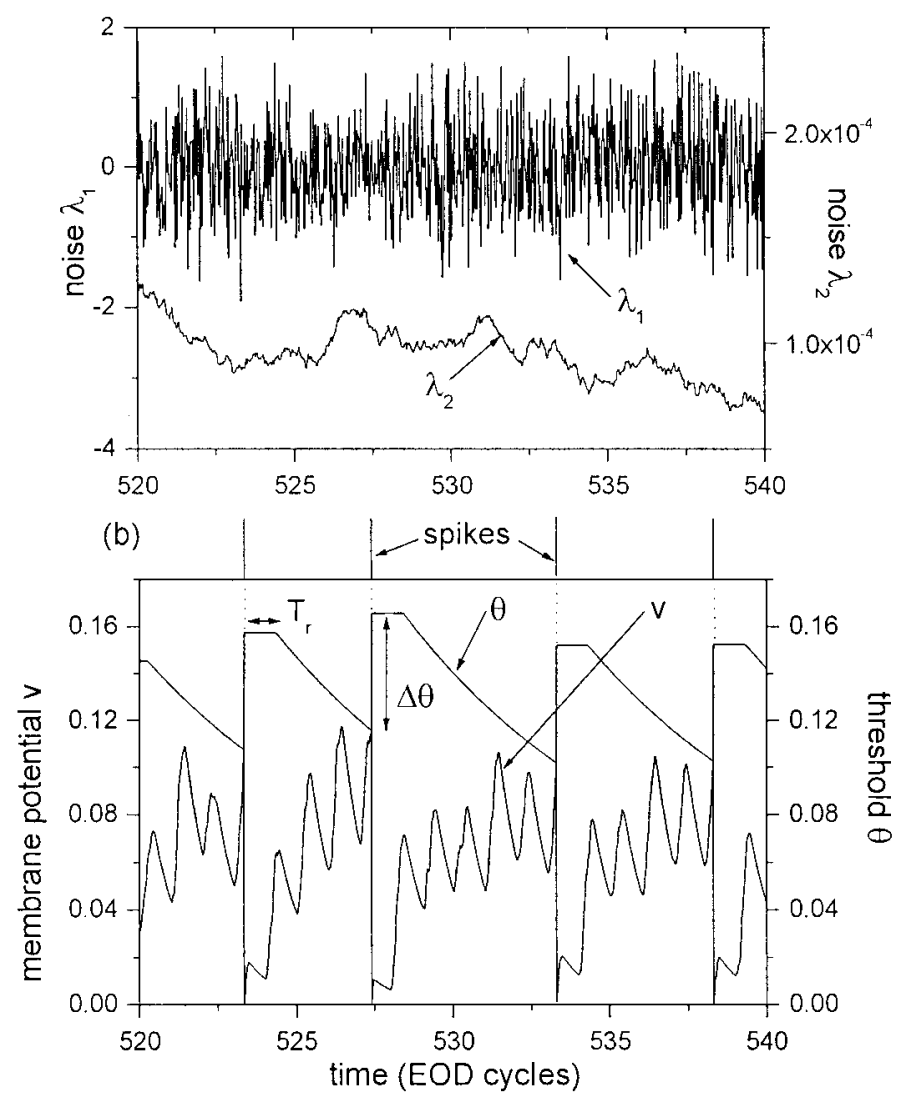

Figure 2. $a$, Noise terms $\lambda_{1}$ (top curve) and $\lambda_{2}$ (bottom curve) as a function of time. $\lambda_{2}$ varies more slowly than $\lambda_{1}$ and is four orders of magnitude smaller. $b$, Voltage (bottom curve) and threshold (top curve) trace obtained with the LIFDT model for baseline activity (no AMs) showing the firing rule. When voltage equals threshold, a spike is said to have occurred, and voltage is reset to zero, whereas threshold is incremented by a constant $\Delta \theta$. The threshold is kept constant to simulate the absolute refractory period $T_{r}$ (equal to one EOD cycle) and then decays exponentially with time constant $\tau_{\theta}$ to its equilibrium value $\theta_{0}$. Parameter values used are given in Appendix A.

$$
\rho_{j}=-0.385 \delta_{1 j}+0.00225 e^{-\frac{j}{4000}},
$$

where $\delta_{i j}$ is the Kronecker delta function $\left(\delta_{i j}=1\right.$ if $i=j$ and $\delta_{i j}=$ 0 if $i \neq j)$. The corresponding spectral density function is plotted in Figure 6. We have only retained the negative SCC at lag 1 because it is dominant. We see that the spectral density function corresponding to the SCCs given by Equation 6 is very similar to the one obtained with our model for $D_{2}=9 \times 10^{-6}$ (EOD cycles $)^{-2}$. Because the SDF and the SCC sequence are completely equivalent (Cox and Lewis, 1966) (see also "Signal detection theory"), this justifies our assumptions. These positive correlations at lags $>1$ sum up according to Equation 1 to give the increase in the Fano factor. Thus, adding a slow additive noise to the membrane voltage increases spike train variability at long counting times. ISI sequences obtained from other neural systems have been shown in some cases to display negative ISI correlations at short lags and positive ISI correlations at long lags (Lowen and Teich, 1992). Furthermore, an increase in the Fano factor curve has been observed in many preparations (Teich, 1992; Lowen and Teich, 1992, 1996; Teich et al., 1996, 1997; Turcott and Teich, 1996) and has been modeled by driving the
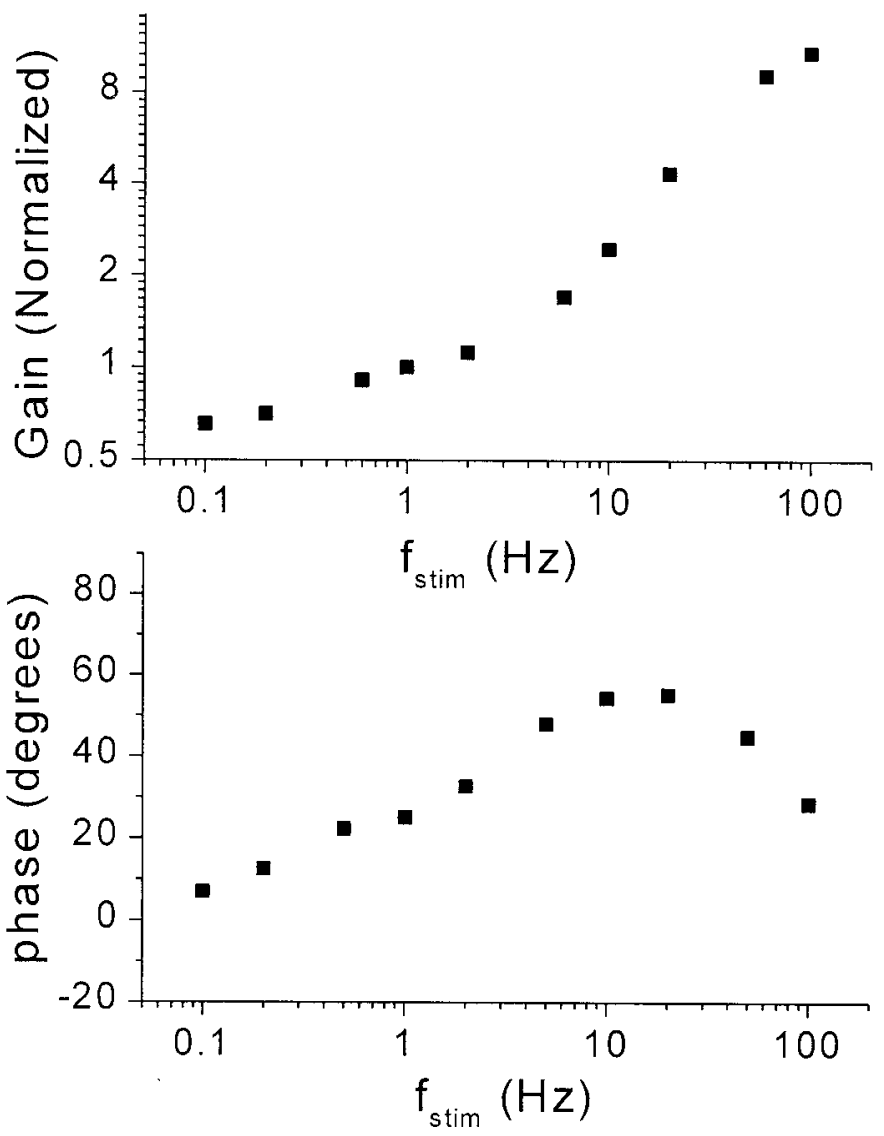

Figure 3. Gain and phase response curves obtained with both models for sinusoidal AMs of various frequencies. The root mean squared baseline transdermal potential is $A_{0} / \sqrt{ } 2=0.566 \mathrm{mV}$, which is in the physiological range (Xu et al., 1996; Nelson et al., 1997; Nelson and MacIver, 1999). As in Nelson et al. (1997), we say that a sinusoidal AM has $0 \mathrm{~dB}$ intensity when it produces a $1 \mathrm{mV}$ change (RMS) in transdermal potential. SAMs of various frequencies were presented to the model with the same intensities used in Nelson et al. (1997) to construct the phase and gain curves. The gains have been normalized by the value 1060 spikes per second per millivolt (this value is in the physiological range) obtained for $f_{\text {stim }}=1 \mathrm{~Hz}$.

rate of a Poisson spike generator with colored noise (Teich, 1992; Teich et al., 1996, 1997). This is a feature of our biophysically plausible simple model of the P-unit. An intuitive explanation of this interesting phenomenon is given in appendix $\mathrm{C}$ while a full explanation is beyond the scope of this paper and will be presented elsewhere.

It is known experimentally that the Fano factor curves obtained for different P-units have the same qualitative shape, although their minimum occurs between 40 and 1000 EOD cycles (Ratnam and Nelson, 2000). Our model produces Fano factor curves quantitatively similar to those obtained experimentally. Furthermore, the location of the minimum is found to be dependent mainly on $D_{2}$; in fact, by a suitable choice of $D_{2}$, we can obtain Fano factor curves matching the full experimental range (data not shown). For example, if we take $D_{2}=10^{-4}$ (EOD cycles $)^{-2}$, then the minimum of the Fano factor curve is at $T=40$ EOD cycles. On the other hand, if we take $D_{2}=10^{-6}$ (EOD cycles $)^{-2}$, then the minimum is at $T=1000$ EOD cycles.

These results imply that the remarkable regularity of P-unit spike trains at counting times of $\sim 250$ EOD cycles (within the experimentally observed range) (Ratnam and Nelson, 2000) can be entirely explained by negative ISI correlations that are present 


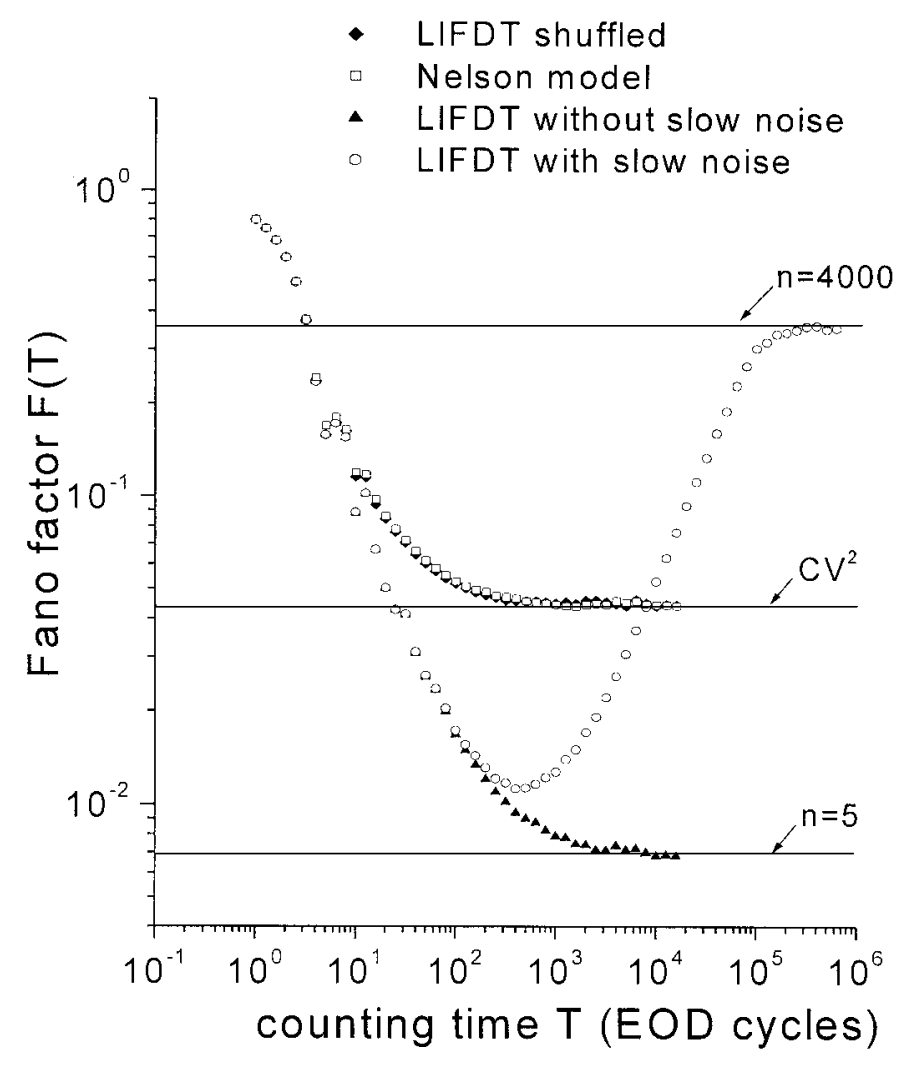

Figure 4. Fano factor curve obtained with the models. The Nelson model has no significant serial correlations amongst ISIs; hence, the Fano factor tends toward the coefficient of variation squared. Random shuffling of the ISI sequence obtained with the LIFDT model removes ISI correlations and gives the same results as the Nelson model. The negative ISI correlations decrease the Fano factor, resulting in a lower asymptotic value. However, adding a weak noise with a long correlation time leads to an increase in the Fano factor at higher counting times (see text and Appendix $\mathrm{C}$ for an explanation).

experimentally and in the LIFDT model at low lags. These arise from cumulative relative refractoriness exhibited by our dynamic threshold. We have shown here that negative SCCs contribute to the reduction of the Fano factor and that positive SCCs increase the Fano factor according to Equation 1. Ratnam and Nelson (2000) have shown that modeling the ISI sequence by a first-order Markov chain gave the correct SCC at lag 1. However, their SCCs at longer lags had higher absolute values than observed experimentally and alternated in sign. As a consequence, the SCC sum for their model was greater (less negative) than the one calculated from the experimental data. Thus, the Fano factor calculated with their Markov chain model did not decrease as much as the Fano factor from their experimental data. In contrast, our model reproduces the descending part of the Fano factor curve seen experimentally [compare our Fig. 4 with Ratnam and Nelson (2000), their Fig. $11 F]$.

We now discuss the power law increase of the Fano factor curve in more detail. We first note that because we are using OrnsteinUhlenbeck noise, which has a finite correlation time $\tau_{2}$, the Fano factor will eventually saturate to a finite value. This value is equal to 0.36188 (Fig. $4, n=4000$ line) and is given approximately by taking the SCCs up to lag 4000 in Equation 1 (hence $\rho_{j}=0$ for $j>$ 4000). This implies that the 4000th ISI is still correlated to the first one. Ratnam and Nelson (2000) found that, for many P-units, the ISI sequence cannot be modeled by a Markov process of order

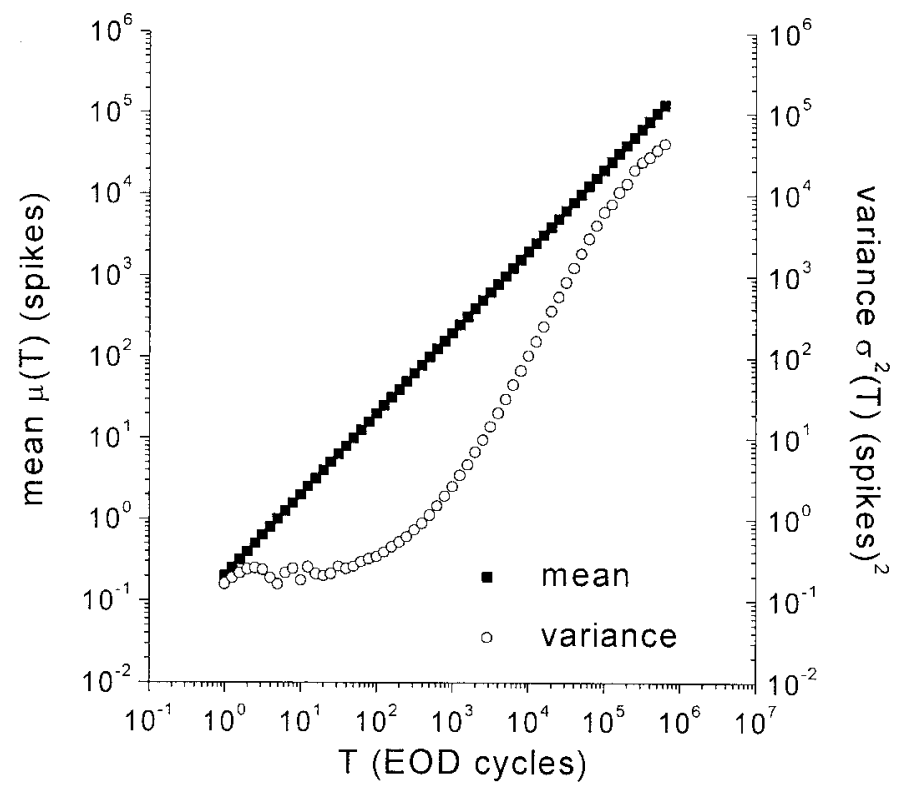

Figure 5. Mean and variance of the PND as a function of counting time $T$ for the LIFDT model with slow noise intensity $D_{2}=9 \times 10^{-6}$ (EOD cycles $)^{-2}$. The mean increases linearly with counting time. The variance is at first almost constant, which leads to a decrease in $F(T)$; it then increases faster than the mean $[F(T)$ increases]. At long counting times, both increase at the same rate; hence $F(T)$ is constant.

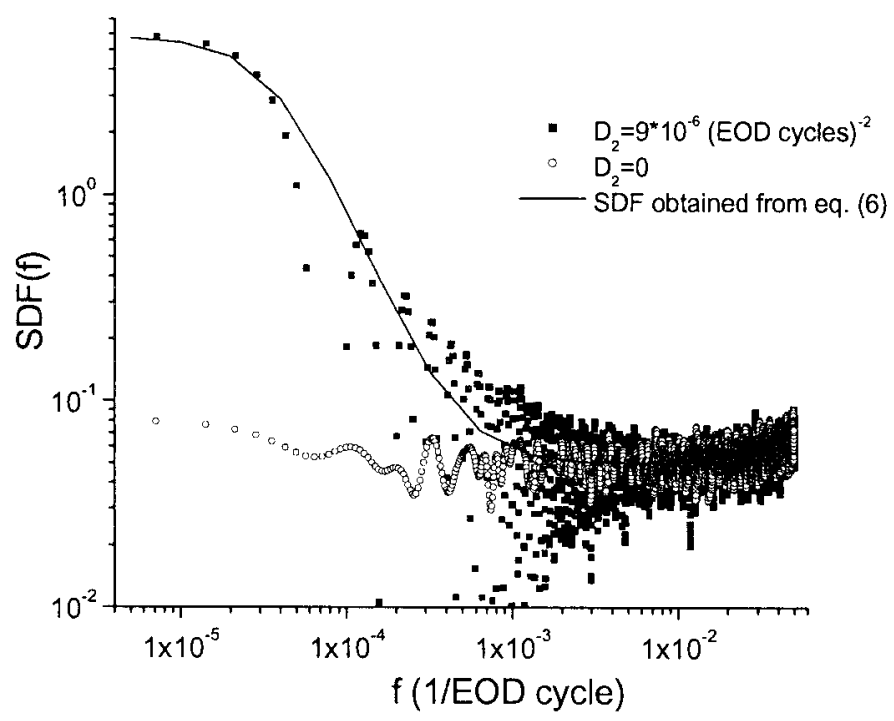

Figure 6. Spectral density function obtained with the LIFDT model with $D_{2}=0$ and $D_{2}=9 \times 10^{-6}$ (EOD cycles $)^{-2}$. Power law behavior is observed with $D_{2}=9 \times 10^{-6}$ (EOD cycles) ${ }^{-2}$ for low frequencies. The spectral density function obtained by assuming a form for the SCCs (see text) is also shown (solid line) and matches the curve obtained for $D_{2}=$ $9 \times 10^{-6}(\text { EOD cycles })^{-2}$.

$<10$. Our results suggest that a Markov chain of ISIs of order at least 4000 would be required to correctly reproduce this feature of the Fano factor curve. In contrast, our simple dynamical model accurately accounts for the full behavior of the Fano factor curve. One plausible origin of the slow Ornstein-Uhlenbeck noise might be fluctuations in synaptic neurotransmitter secretion rates that exhibit long-term correlations (Lowen et al., 1997). However, it could also be caused by slow drifts in EOD amplitude or frequency (Moortgat et al., 1998). 

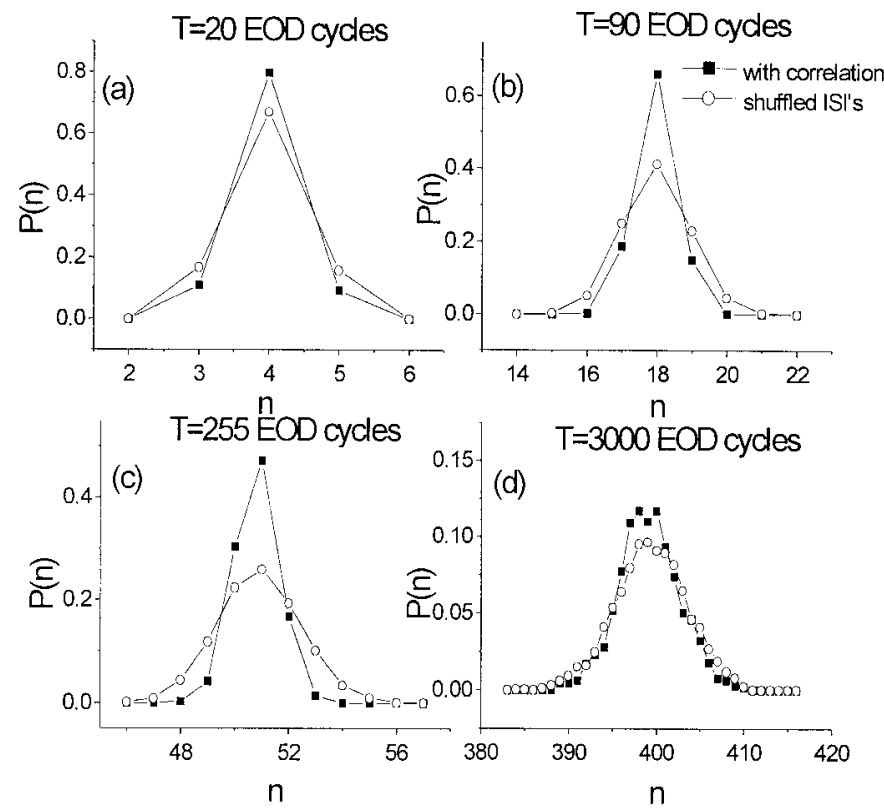

Figure 7. PNDs obtained for both models for various counting times: $(a)$ 20, (b) 90, (c) 255, (d) 3000 EOD cycles. ISI correlations reduce the variance of the PND while keeping the mean unchanged. This effect is maximal at counting times in which the Fano factor is minimal.

\section{Pulse number distributions and ROC curves}

We show in Figure 7 the PNDs that were obtained in the presence and absence of ISI correlations for four different counting times for the LIFDT model. We see that ISI correlations have minimal effects at short counting times such as 20 EOD cycles (Fig. 7a). The effect of negative ISI correlations increases with counting time (Fig. 7b). It is very important around 255 EOD cycles (Fig. 7c), at which the variance of the PND is reduced while the mean is left unchanged. This effect diminishes for longer counting times (Fig. $7 d$ ) at which positive ISI correlations contribute to the broadening of the PND.

This has implications for weak signal detection using the ideal observer paradigm (Green and Swets, 1966). Intuitively, if $P_{0}(n, T)$ and $P_{1}(n, T)$ do not overlap much, then we have a very good detector (Gabbiani and Koch, 1998). As mentioned previously, a good measure for this is the discriminant measure $d$ (Green and Swets, 1966; Nachimas, 1972; Snippe and Koenderink, 1992) defined by:

$$
\mathrm{d}=\frac{\left|\mu_{1}-\mu_{0}\right|}{\sqrt{\sigma_{1}^{2}+\sigma_{0}^{2}}},
$$

where the vertical bars denote the absolute value and $\sigma_{i}^{2}$ and $\mu_{i}$ are the respective variance and mean of $P_{i}(n, T)$. We have assumed in Equation 7 that the $P_{i}(n, T)$ are Gaussian (i.e., we have neglected their third and higher moments). This is not too restrictive as they approach Gaussian distributions for high $T$ by the central limit theorem. Furthermore, the PNDs obtained with the models are bell shaped (Fig. 7), and the Gaussian approximation is reasonable. Optimal discrimination, and hence detection, requires $d$ to be higher than some threshold $d_{\text {crit }}$. Let $f_{0}$ be the baseline steady-state firing rate of the electroreceptor and suppose that the stimulus varies slowly with time and leads to a new steady-state firing rate $f_{1}$. Here, we do not consider transients in P-unit firing rate that can occur after a change in EOD amplitude

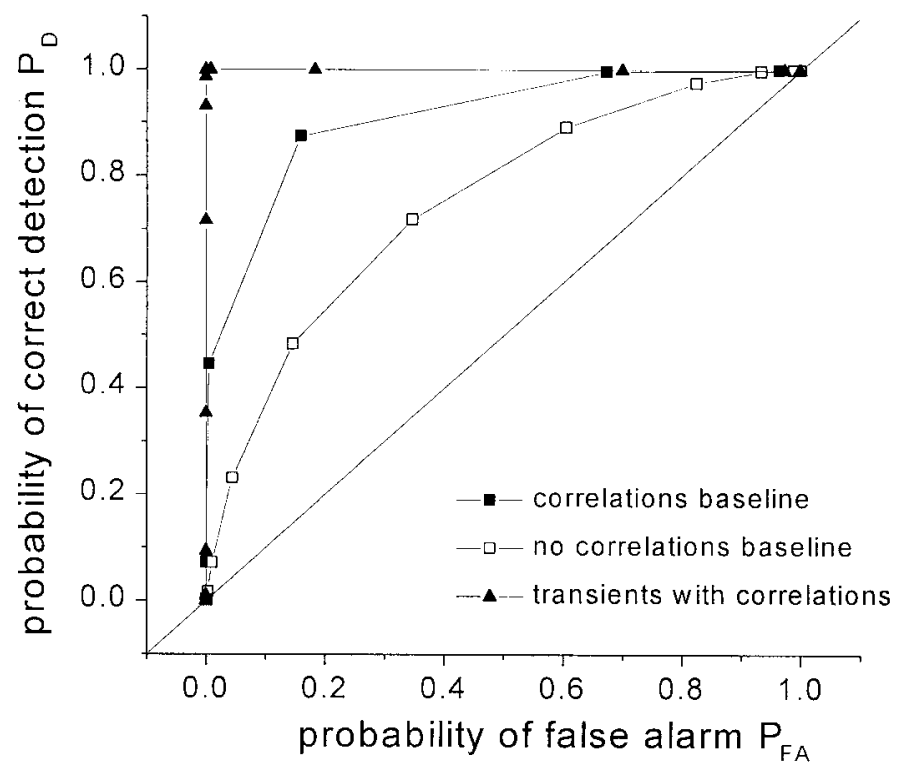

Figure 8. ROC curve obtained for both models. Correlations improve the ROC curve by decreasing the variance of the PNDs, leading to a better discriminability between the distributions. The stimulus was a $4 \%$ step increase in EOD amplitude (the new amplitude value is equal to 1.04 times the old value). Including the transients leads to a further increase of the mean of the PND with stimulus, which further increases discriminability and, hence, improves further the ROC curve.

(Xu et al., 1996; Nelson et al., 1997), but rather only the new steady-state firing rate. Furthermore, suppose that the signal is weak, and that as a consequence, the variances of the PNDs are approximately equal. Hence, $\mu_{1}(T)=\left(f_{1} / f_{0}\right) \mu_{0}(T)$ and $\sigma_{1}^{2}(T) \cong$ $\sigma_{0}^{2}(T)$ and using Equation 7 , the inequality $d \geq d_{\text {crit }}$ becomes:

$$
F(T) \leq\left|\frac{f_{1}}{f_{0}}-1\right| \frac{\sigma_{0}(T)}{\sqrt{2} d_{\text {crit }}},
$$

because $F(T)=\sigma_{0}^{2}(T) / \mu_{0}(T)$. Furthermore, $\sigma_{0}(T)$ does not vary much if we consider low counting times (Fig. 5), hence it can be considered constant to a first approximation. From Equation 8, the lower the Fano factor, the better the detector. A good value for $d_{\text {crit }}$ that gives almost no overlap between the $P_{0}(n, T)$ and $P_{1}(n, T)$ distributions is three. Using this value, one can find a lower bound for $\left|\left(f_{1} / f_{0}\right)-1\right|$ from Equation 8 . From the data for $T=255$ EOD cycles, $\sigma_{0}(255)=0.78, F(255)=0.012$, hence a single P-unit can near perfectly discriminate steady-state response to slow amplitude modulations as low as $6.5 \%$ from baseline firing. The negative ISI correlations at low lags make the PNDs narrower and lead to a significant improvement in the ROC curve (Fig. 8). These fish are very good at detecting prey using their electrosensory system (Nelson and MacIver, 1999). Because there are relatively few numbers of false strikes (Nelson and MacIver, 1999), $P_{\text {FA }}$ must be low (Ratnam and Nelson, 2000). Our results show that the improvement in the detection probability $P_{\mathrm{D}}$ attributable to ISI correlations is in fact greatest for low $P_{\text {FA }}$. Thus, the animal significantly improves its chances at detecting prey by having negatively correlated ISIs.

In the above analysis, we did not consider the effect on signal detection of transients in firing rates that are associated with changes in EOD amplitude (Xu et al., 1996; Nelson et al., 1997). Transients will help the animal in detecting weak signals by increasing or decreasing the firing rate, thus shifting the mean of 
the PND with stimulus away from the baseline PND. This is confirmed in Figure 8 in which the transients resulting from a $4 \%$ step increase in EOD amplitude lead to a near perfect detection of the signal. These transients are present in both models because both incorporate the filter given by Equations 3-5. A full exploration of their effects will be done elsewhere.

Thus, there are two phenomena at work here: transients resulting from a change in EOD amplitude will shift the mean of the PND, whereas negative correlations will reduce the variance. The combined effect is better discriminability between the PNDs, hence a better ROC curve and a lower stimulus contrast threshold for signal detection.

Usually, fluctuations caused by noise average out over time, and the ROC curve improves (Gabbiani and Koch, 1998). In our case, the ROC curve becomes worse at longer counting times as the positive ISI correlations increase variability (Fig. 7d). Thus, there is a time window in which signal detectability is optimal for the animal, and it corresponds to the counting time at which the Fano factor is minimal. This optimal counting time has been shown to vary between 40 and 1000 EOD cycles (Ratnam and Nelson, 2000) within the P-unit population.

\section{Entropy}

All the previous analysis assumed a rate code, and thus spike timing was considered unimportant. A recent study on the electroreceptors of a very similar electric fish (Kreiman et al., 2000) showed that significant jitter could be added to the spike train without affecting the quality of encoding by using the stimulus reconstruction technique (Rieke et al., 1997; Gabbiani and Koch, $1998)$ when the stimulus cutoff frequency was low $(<20 \mathrm{~Hz})$. This suggests that a rate code might be more relevant for the encoding of low-frequency stimuli.

However, electrocommunication signals contain much higher frequency components (Metzner and Heiligenberg, 1991; Zupanc and Maler, 1993; Dulka et al., 1995). In fact, the animal can detect AM frequencies $>200 \mathrm{~Hz}$ (Bastian et al., 2001). We use information theoretic measures to assess the quality of encoding time, varying stimuli by P-units at such frequencies. In particular, we will assess the role of ISI correlations by comparing the information rates obtained with and without their presence.

To assess the ability of the P-unit to encode different frequency stimuli, we used low-pass filtered Gaussian white noise with a variable cutoff frequency $f_{c}$ (Wessel et al., 1996). The same stimulus was presented to both models, and the resulting baseline and noise entropies for word lengths up to 16 were calculated. Because the unit can fire at most once per EOD cycle, it is natural to make the bin size $\Delta \tau$ equal to one EOD cycle. We have also plotted results obtained with a binomial process (each bin has probability $p$ of being assigned the value 1 and probability $1-p$ of being assigned the value $0, p$ being the probability of firing per EOD cycle equal to 0.2 in our case). For such a process, the entropy of words of length $L$ as a function of $L^{-1}$ is constant (Shannon, 1948; Cover and Thomas, 1991); this permits us to verify the accuracy of our algorithm. The calculated entropy for the binomial process was significantly lower than the true value (Fig. 9) for words of length $>6$. This is attributable to the finite length of the spike train that is considered, which leads to undersampling for words of longer lengths (Strong et al., 1998). We thus only considered words up to length 6 (i.e., $1 / L$ goes from 1 to $1 / 6$ ). We plot the baseline entropies obtained for each model in Figure 9. Because a clear deviation from linearity can be seen in each case, we performed a quadratic fit according to Equation 2. Note

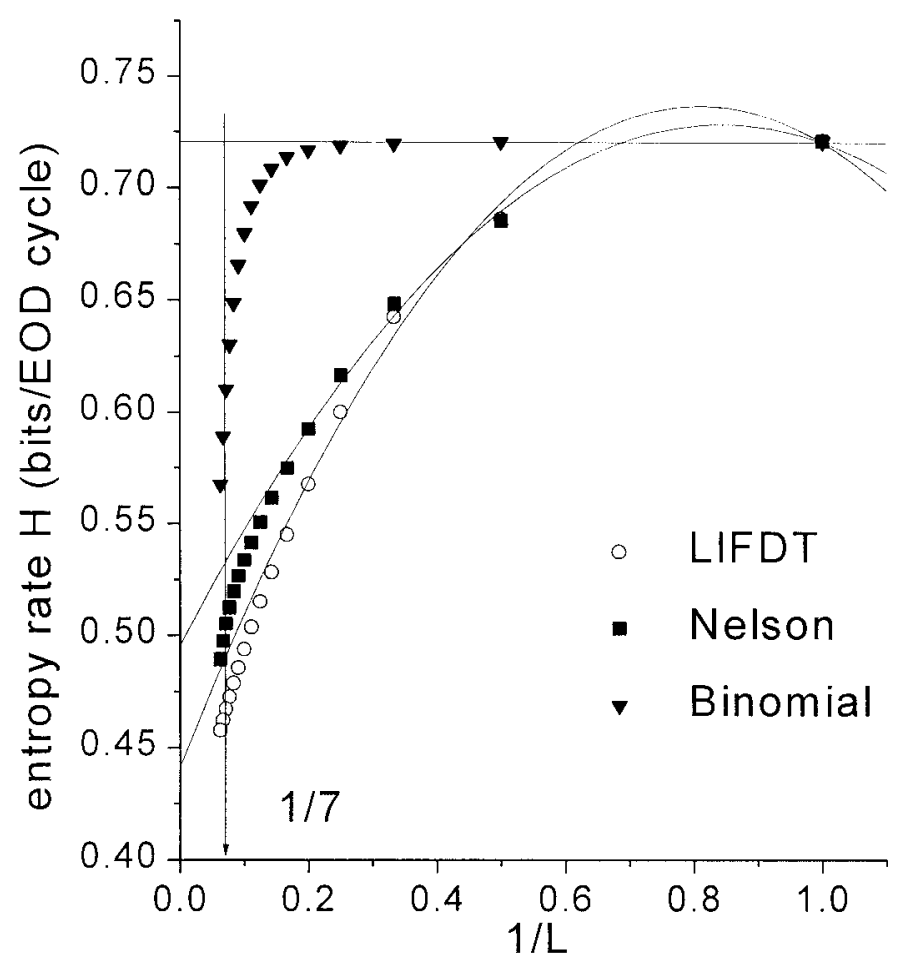

Figure 9. Baseline entropies of words of length $n$ calculated from 1000 realizations of duration 10,000 EOD cycles each for both models. Comparison with a binomial process for which these entropies should be constant is shown to verify the accuracy of the results. Finite sampling errors occur for words of length $>7$ and lead to results that are lower than the true value (Strong et al., 1998). We thus take only the results for words up to $n=6$ for the fits. Clear deviation from linearity is still seen for the first few points in both cases. Entropies are lower for the LIFDT model due to correlations. Also shown are the best fits obtained for the data.

that the baseline entropy values for our model are always lower than for Nelson's as correlations reduce entropy (Shannon, 1948; Cover and Thomas, 1991). However, noise entropies will also be lower for our model for the same reason. It is thus not clear a priori what effect correlations will have on information transfer.

We thus calculated the information rates at different stimulus contrasts $\sigma_{\text {stim }}$ for both models. The baseline and noise entropy rates for different contrasts were calculated at a fixed cutoff frequency $f_{c}=100 \mathrm{~Hz}$ to assess the capacity of the P-unit to encode high-frequency stimuli (as discussed above). We plot in Figure 10 the information rate for both models. As expected, it increases with stimulus contrast for both models; however, the information for the LIFDT model is always higher than the one obtained for Nelson's model. For example, for stimulus contrasts between 0.04 and 0.05 $\mathrm{mV}$, the gain in information rate is approximately 0.04 bits per EOD cycle, which corresponds to 40 bits per second. For both models, the correlation between successive spikes in the presence of a stimulus increases with $\sigma_{\text {stim }}$, resulting in a decrease in noise entropy rate. This leads to an increase in the information rate. However, this correlation is higher for the LIFDT model because of the dynamic threshold, which reduces the noise entropy even more and leads to a higher information rate.

We now look at the dependence of information rate on stimulus cutoff frequency $f_{c}$. We thus construct an "information tuning curve" for the two models (Fig. 11a), i.e., the dependence of information rate on stimulus cutoff frequency. For both models, information rates are small at low cutoff frequencies and increase 


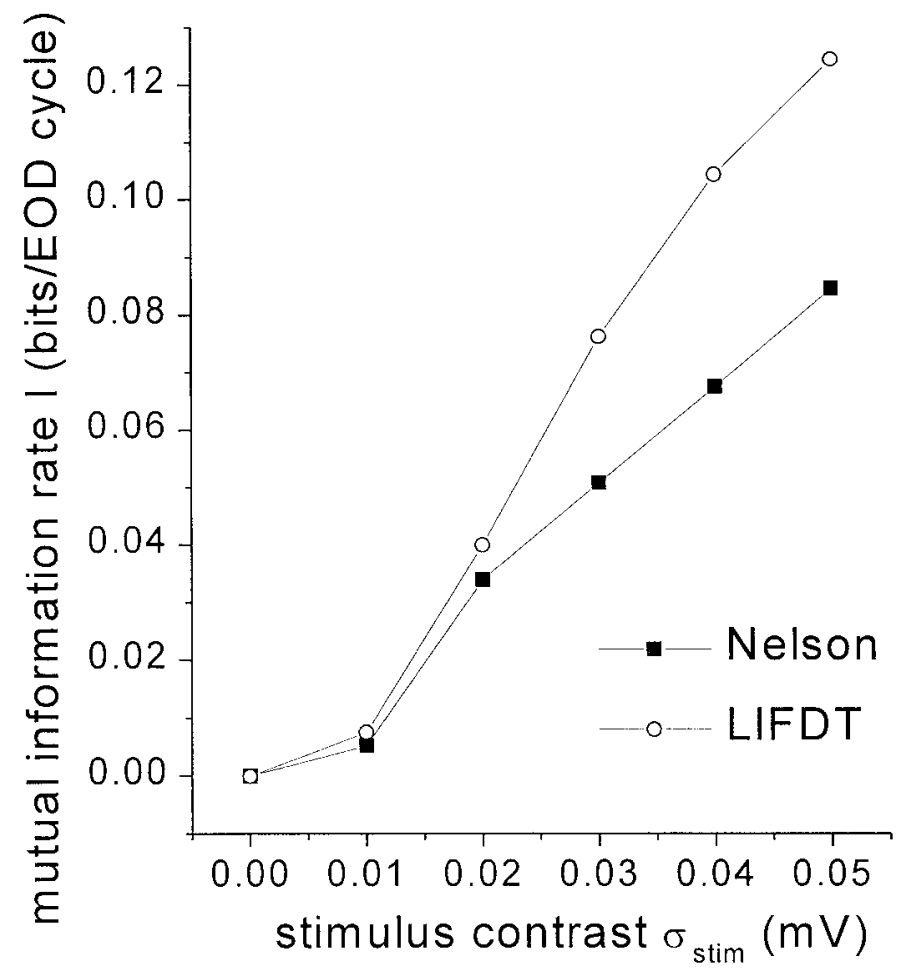

Figure 10. Information rate as a function of stimulus contrast for both models. The stimulus is low-pass filtered Gaussian white noise with cutoff frequency $100 \mathrm{~Hz}$ and has mean of zero and $\mathrm{SD} \sigma_{\text {stim. }}$. Information rate increases with contrast for both models, but it is higher in the LIFDT model than in the Nelson model.

for higher cutoff frequencies, such as those near the mean baseline firing rate of the neuron $(200 \mathrm{~Hz}$ in our case). The general shape is attributable to the fact that the noise entropy decreases as a function of $f_{c}$ (over the range of interest). This decrease may be attributable in part to the high-pass filter characteristics of both models (Eqs. 3-5) over the range of frequencies considered. A more complete analysis of this result will be presented elsewhere.

Information rates were higher for the LIFDT model, compared with the Nelson model over the full range considered. A surprising result is the fact that the gain in information caused by ISI correlations exhibits a maximum at frequencies $\sim 100-150 \mathrm{~Hz}$ (Fig. 11b). Hence, our dynamic threshold enhances the information tuning curve, and this effect is maximal for stimuli with a cutoff frequency $f_{c}$ of the order of the inverse of the decay time constant of the dynamic threshold $\tau_{\theta}$. This effect can be understood intuitively as follows: cumulative relative refractoriness leads to a less variable neural response to repeated stimuli; if the unit fires on the rising phase of the stimulus, then it has time to recover and fire on the next rise of the stimulus, which occurs on average at least after one correlation time $f_{c}^{-1}$. One thus expects this effect to be maximal when $f_{c}^{-1}$ is on the order of the time constant of the cumulative relative refractoriness (modeled here by our dynamic threshold). If $f_{c}$ is too low, then the dynamic threshold does not enhance information transmission, because the stimulus dynamics occur on a much longer time scale. Hence the information rates obtained with both models are approximately the same (Fig. $11 a$, less than $f_{c}=50 \mathrm{~Hz}$ ). For $f_{c}$ high, the dynamic threshold cannot follow the fast stimulus variations, and the two information rates are again the same. There is thus a

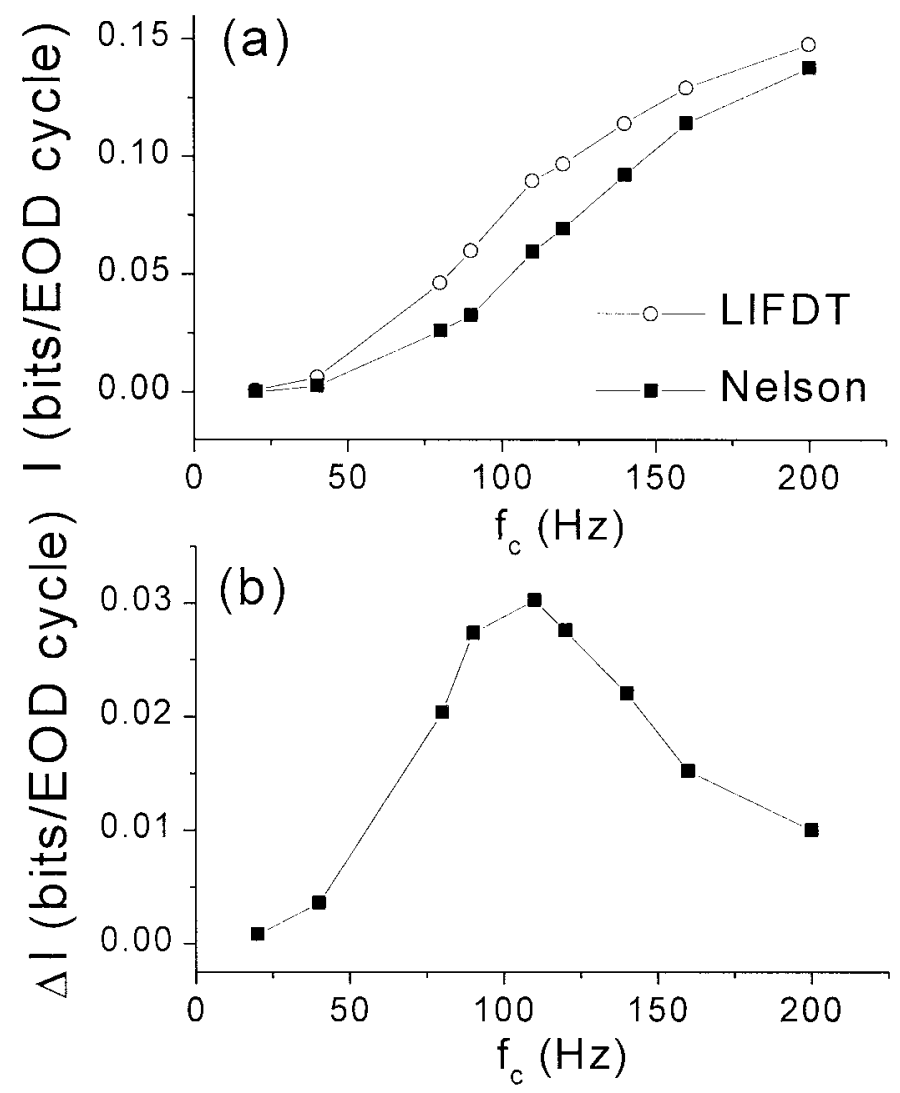

Figure 11. a, Mutual information rate as a function of stimulus cutoff frequency. The stimulus contrast was $\sigma_{\text {stim }}=0.03 \mathrm{mV}$. The information rate increases with frequency for both models. However, information rate is higher for the LIFDT model than for the Nelson model. $b$, Difference between the information rate for the LIFDT model and the Nelson model $\Delta I$ as a function of $f_{c}$. This quantity displays a resonance for frequencies on the order of the inverse of the decay time constant of the dynamic threshold $\tau_{\theta}$.

resonance in the gain in information rate at $\sim 100 \mathrm{~Hz}$ because of cumulative relative refractoriness. The maximum gain is $\sim 0.03$ bits per EOD cycle, which corresponds to 30 bits per second.

Behavioral experiments have demonstrated that the animal can reliably detect AMs with $100-200 \mathrm{~Hz}$ frequencies and that this might be relevant for courtship behavior (Bastian et al., 2001). We hypothesize that the resonance in the P-unit information tuning curve caused by our dynamic threshold may be responsible for this observed sensitivity.

Note that if the stimulus correlation time $f_{c}^{-1}$ is high, then one might expect that the information rate over an EOD cycle would be lower than the one obtained if $f_{c}^{-1}$ was low. This is attributable to oversampling. Note also that for a stimulus cutoff frequency $f_{c}$, the electrosensory system might integrate the input using a time window proportional to $f_{c}^{-1}$. These possible effects can be eliminated by dividing the information rate by $f_{c}$. This results in an estimate $\left(I_{c}\right)$ of the average information transmitted during a time window equal to the correlation time $f_{c}^{-1}$ of the stimulus. This quantity is shown to increase as a function of $f_{c}$ for both models (Fig. 12a) but saturates for high $f_{c}$. Thus, more information is transmitted about high-frequency stimuli as compared to low ones, even when the integration times are normalized. This occurs because the increase in $I_{c}$ is limited by the sampling rate allowed by the baseline firing rate of the P-unit (Nyquist theo- 

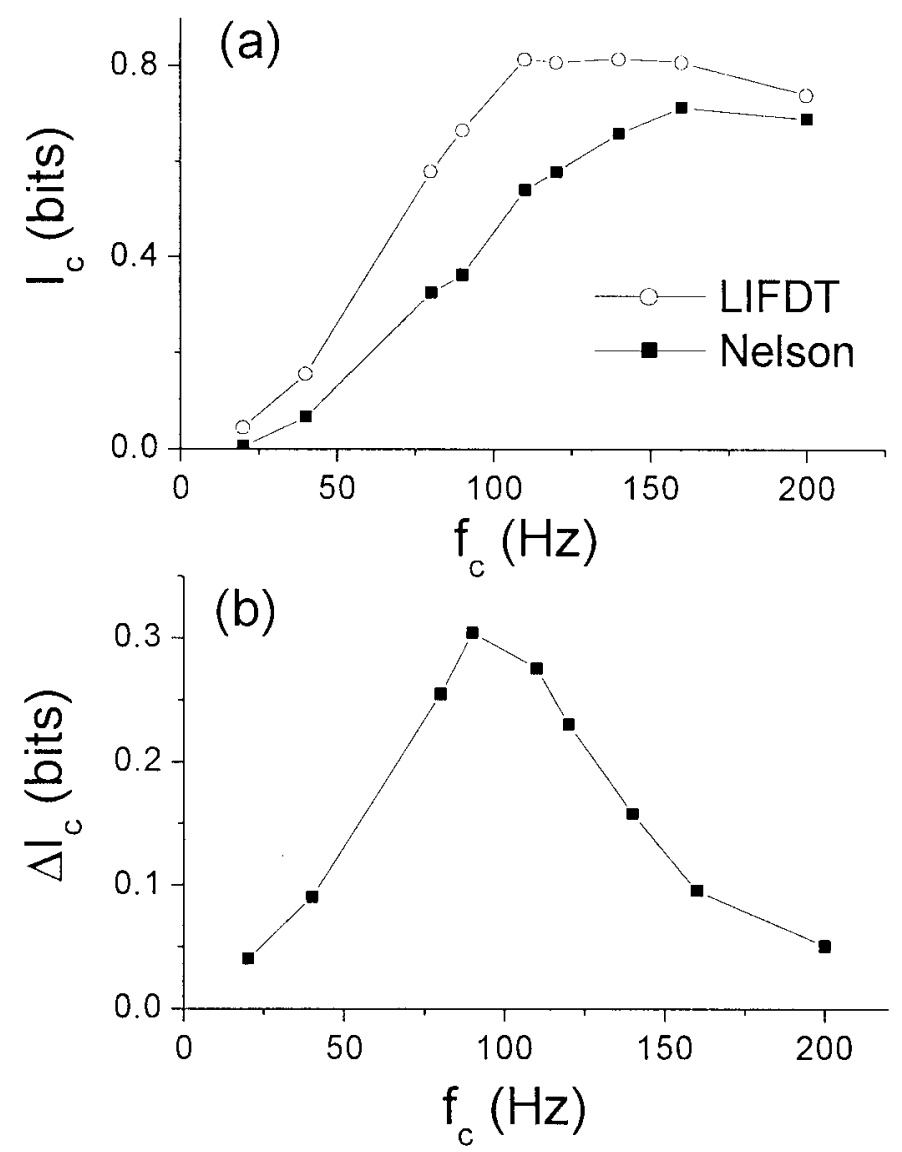

Figure 12. Average information transmitted $I_{c}$ during a time window whose length is equal to the stimulus correlation time $f_{c}^{-1}$ as a function of cutoff frequency $f_{c}$. This quantity increases with $f_{c}$ (see text for explanation).

rem). However, $I_{c}$ is still higher for the LIFDT model. The gain also has a resonance with the maximum $\sim 100 \mathrm{~Hz}$ (Fig. 12b).

\section{Effect of blurring on information rate}

Finally, we show that the importance of spike timing increases with stimulus cutoff frequency. We introduce "blurring" in the spike train by making the bin width $\Delta \tau$ greater and studying the incurred loss in potential information for different cutoff frequencies of the stimulus. We see that this loss is greater for higher cutoff frequencies (Fig. 13). This suggests that spike timing has minimal contributions in encoding information about slowly varying stimuli. Furthermore, this finding agrees with the fact that significant jitter does not affect the quality of the reconstructed stimulus for low stimulus cutoff frequencies (Kreiman et al., 2000). However, spike timing is important for high-frequency time-varying stimuli, and our results show in fact that electroreceptors can encode stimuli that vary on time scales at least as fast as 5-10 EOD cycles. This agrees with the results from a previous study that found that spike timing jitter in electroreceptors was on the order of one to two EOD cycles (Kreiman et al., 2000). This also justifies our use of PNDs for low-frequency stimuli.

\section{Contrasting signal detection and information theory}

As the fish swims by a prey, it will experience a small change in transdermal potential in a time window of $\sim 200$ EOD cycles (Nelson and MacIver, 1999). However, information rates are almost zero for such low cutoff frequencies (this corresponds to

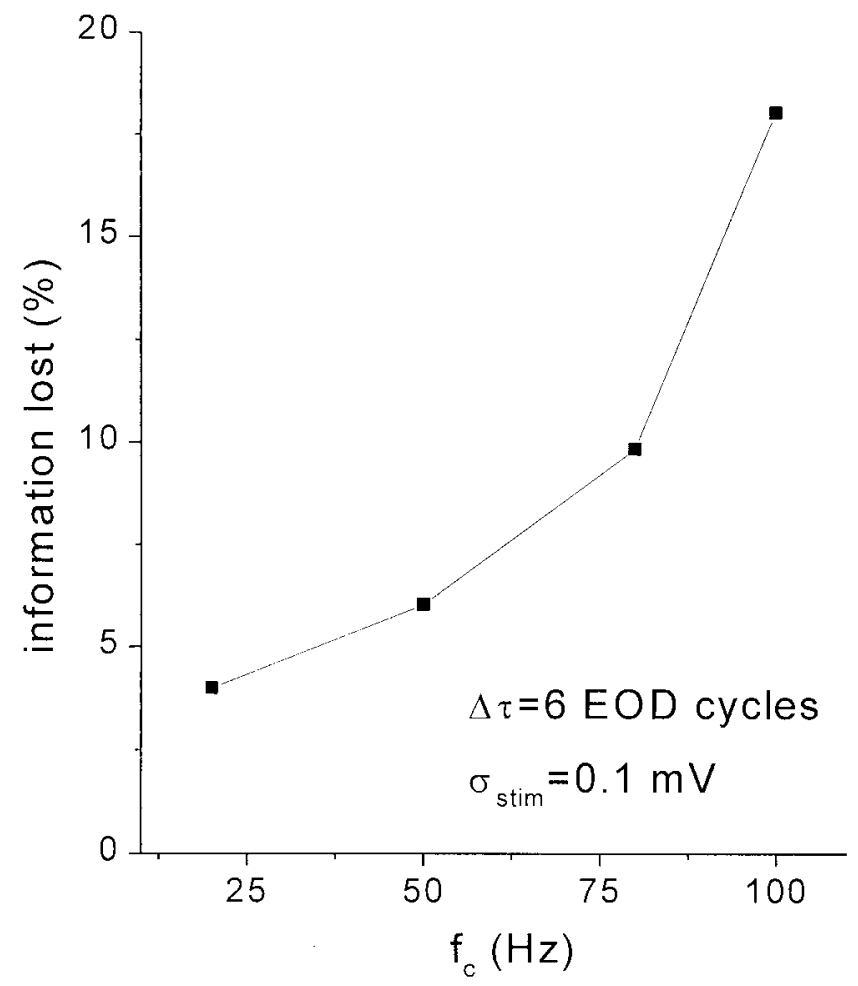

Figure 13. Information loss caused by blurring the spike train. We counted the number of spikes that occurred in successive time intervals of six EOD cycles in length and computed the information rate for that spike train. We can see that fractional loss of information increases with $f_{c}$.

frequencies of $\sim 5 \mathrm{~Hz}$, the information rate is almost zero for $f_{c}<$ $50 \mathrm{~Hz}$ for the particular P-unit cell modeled here) (Fig. 11a). The fact that the animal can readily detect these signals (Nelson and MacIver, 1999) suggests that the mutual information rate that was calculated using baseline entropy is poorly suited for coding of these low-frequency stimuli. This is also the case after the correlation time of the stimulus has been taken into account (Fig. 12a). However, we have shown that measures based on signal detection theory were adept at quantifying the ability of the electroreceptor to transmit information about this type of stimuli (Figs. 7, 8). It is thus more natural to analyse slow time-varying stimuli by looking at the trial-to-trial variability of the PND.

We use a weak, slow time-varying stimulus $\left(\sigma_{\text {stim }}=0.01 \mathrm{mV}\right.$, $\left.f_{c}=1.96 \mathrm{~Hz}\right)$ and look at the PND in a time window of $255 \mathrm{EOD}$ cycles. The portion of stimulus used is shown in Figure 14a. The PNDs obtained with both models are shown in Figure $14 b$. Note that for each model, the PNDs with and without stimulus have almost the same variances. Thus, the main factor for discriminating between the two distributions is the difference in their means. This is not captured by entropy measures because they do not depend on the mean of the distribution used (Shannon, 1948).

However, as shown previously (Fig. 7), the variance of the PNDs that were obtained with the LIFDT model are smaller than those obtained with the Nelson model. Note that the means are separated by as few as three spikes in both cases (as low as $6 \%$ difference). The lesser overlap for the LIFDT model results in a dramatic improvement in discriminability. For example, the probability of obtaining 53 spikes for the LIFDT model is 0.3 with stimulus and 0.05 without stimulus, corresponding to a ratio of 6 . For the Nelson model, this ratio is 2.5. Our results thus show that a single P-unit could discriminate as few as two extra spikes in a 

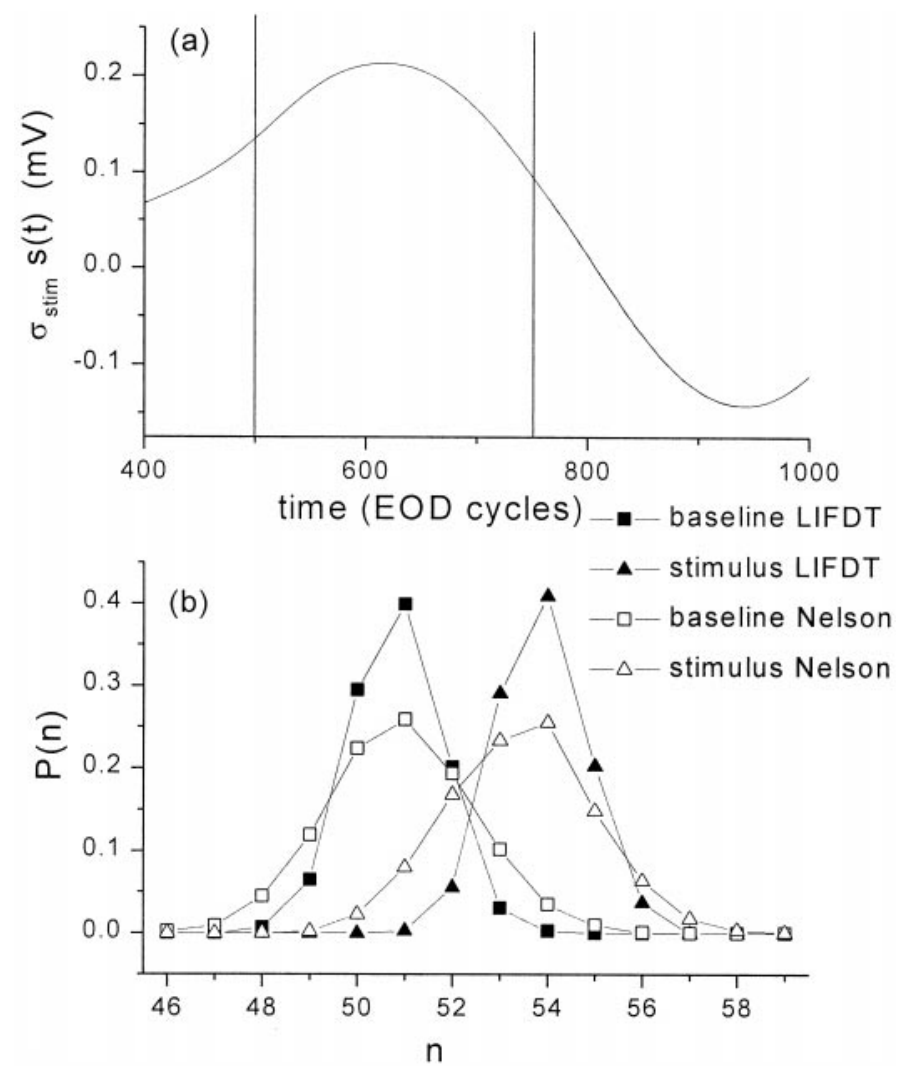

Figure 14. a, A realization of low-pass filtered Gaussian white noise with cutoff frequency $f_{c}=1.96 \mathrm{~Hz}$ and contrast $\sigma_{\text {stim }}=0.01 \mathrm{mV}$. The counting time window is between the horizontal lines (from 500 to 755 EOD cycles). $b$, PND obtained with this portion of stimulus as compared with the one obtained from baseline dynamics. The variances of the PNDs are nearly identical and low, whereas the means are very different, which leads to a good discriminability $d$. The difference between the means is not captured by entropy measures, because they do not depend on the mean and also assume infinite spike trains. Because there is no averaging over the stimulus in this case, signal detection yields better results than information theory.

time window of 255 EOD cycles as already suggested by Ratnam and Nelson (2000). Their first-order Markov process was not able to reproduce the experimentally observed probabilities of correct detection. This is because the Fano factor for their model was higher than the one for the experimental data. The key factor in this remarkable sensitivity is the negative ISI correlations that were observed experimentally and that result from cumulative relative refractoriness exhibited by our simple model with dynamic threshold.

\section{DISCUSSION}

Using two models, one with baseline ISI correlations and one without, we have shown that negative ISI correlations that are present experimentally play an important role in the animal's ability to detect both slowly and rapidly time-varying stimuli. A first analysis based on signal detection theory, which assumes rate coding, revealed that the ISI correlations dramatically enhanced the detectability of low-frequency weak signals. We then used information theory to quantify the ability of the afferents to encode time-varying stimuli with various cutoff frequencies. We found that ISI correlations also helped in this case and that the effect was maximal for cutoff frequencies on the order of the inverse of the decay time constant of our dynamic threshold that was used to include cumulative relative refractoriness. By comparing both approaches, our study suggests that a rate code can be assumed for low-frequency stimuli, whereas spike timing is important for high-frequency signals.

\section{Comparison of models}

Our previous simple model (Chacron et al., 2000) reproduced baseline first- and second-order ISI statistics that were seen experimentally, such as the ISI correlations. Here, this model was extended to include experimentally measured linear response properties to sinusoidal AMs by Nelson et al. (1997). To quantify the effects of correlations, we used a second model proposed by Nelson et al. (1997) that gave identical first-order statistics and responses to sinusoidal AMs, except that there were no ISI correlations.

\section{Spike train variability and signal detection}

Spike train variability as measured by the Fano factor was computed for both models at various time scales. Although able to reproduce first-order ISI statistics as well as responses to AMs, the Nelson model did not reproduce experimentally observed spike train variability as measured by the Fano factor at counting times $>10$ EOD cycles (Fig. 4) (Ratnam and Nelson, 2000). The asymptotic value of the Fano factor for the Nelson model was shown to equal the square of the coefficient of variation of the ISIH as expected from Equation 1 in the absence of ISI correlations. The Fano factor obtained from our LIFDT model also tends toward this value when the ISI sequence is randomly shuffled to eliminate ISI correlations, thus proving that the Nelson model did not display any significant ISI correlations.

The negative ISI correlations obtained with our simple LIFDT model bring the Fano factor down (Cox and Lewis, 1966) (Eq. 1) to experimentally determined values for longer counting times; a discrepancy was, however, observed for counting times $>150$ EOD cycles as the Fano factor decreased monotonically instead of increasing. To get this increase in variability at long counting times, it was necessary to add a weak noise with a long correlation time to the model dynamics. This noise had no effect at low counting times, as explained in "Fano factor" and in Appendix C. However, it led to positive ISI correlations extending to long lags as seen by the increase of the spectral density function at low frequencies (Fig. 6). The positive ISI correlations were weak, decayed exponentially up to long lags, and summed up according to Equation 1 to give the increase in the Fano factor. This increase has been observed in many neurons and may be of synaptic origin or attributable to drifts in EOD amplitude or frequency. These ISI correlations make modeling of electroreceptors by Markov chains of ISIs difficult because ISI correlations can extend out to lags in the thousands. However, the addition of an extra noise term in our simple model gave quantitatively accurate results and could be used to incorporate these effects into other neuron models.

We used the ideal observer paradigm (Green and Swets, 1966) to optimally discriminate between pulse number distributions with and without stimulus (Gabbiani and Koch, 1998). It was shown that negative ISI correlations reduced the variance of these pulse number distributions without changing their means significantly, hence increasing their discriminability. This led to an improvement in the receiver operating characteristic curve that was maximal at counting times at which the Fano factor was minimal. A criterion for near perfect discrimination was derived, and it was shown that signals leading to a $6.5 \%$ increase in firing 
rate would be discriminated in this manner on the basis of steady-state dynamics alone. Transients caused by the filter further lowered this detection threshold by increasing or decreasing the firing rate computed over the duration of the stimulus, thus leading to a further increase in discriminability. Signal detection analysis is not appropriate for zero mean high-frequency signals (e.g., beat frequencies generated by fish with high EOD frequency differences) because spike train variability is high at low counting times (Softky and Koch, 1993; Borst and Theunissen, 1999). It is, however, appropriate for low-frequency signals such as those caused by prey.

\section{Using information theory to quantify the coding of time-varying stimuli}

The trial-to-trial variability of the neural response to a repeated stimulus was characterized by the noise entropy rate. The entropy rate of the baseline spike train was estimated, and the difference between baseline and noise entropy rates was used to quantify the information transfer rate about the stimulus. This definition is natural in our case because we have baseline firing activity and signal detection must be based on a change from this baseline activity (Ratnam and Nelson, 2000). We compared the information rates obtained with our model and Nelson's. It was shown that the information rate increased in both cases with stimulus contrast. This agrees with the fact that the electroreceptor is better at encoding stronger stimuli (Wessel et al., 1996). However, negative ISI correlations could help the electroreceptor in the coding of fast time-varying stimuli because information rates computed with our model were higher. This is caused by the fact that correlations reduce the noise entropy even more than the baseline entropy. We note that our results are consistent with previous studies that have demonstrated that a refractory period can improve the linear correlation between the stimulus and the instantaneous firing rate (Chialvo et al., 1997) as well as the neural precision (and hence the mutual information) (Berry and Meister, 1998). However, the models did not incorporate ISI correlations. Furthermore, in Berry and Meister (1998), the increase in refractory period led to a decrease in mean firing rate, and the causes of the increase in mutual information were not clear. In our analysis, we examined the effect of ISI correlations on mutual information and signal detection without concomitant changes in the mean firing rate.

Information rates as a function of stimulus cutoff frequency were also computed. Our results show that the information rate increased with cutoff frequency as expected from the high-pass characteristics of $\mathrm{P}$-afferents (Xu et al., 1996). Information rates were higher for the LIFDT model, and the gain attributable to correlations exhibited a resonance with a maximum of $\sim 100 \mathrm{~Hz}$. This frequency corresponds to the inverse of the decay time constant of our dynamic threshold. Remarkably, there is evidence that these fish respond preferentially to electrocommunication signals with a frequency content of $\sim 100 \mathrm{~Hz}$ (Bastian et al., 2001).

\section{Comparing signal detection and information theory}

Finally, we have shown that the loss of information about the stimulus incurred by decreasing the spike-timing resolution increases with cutoff frequency (Fig. 13). This suggests that spike timing is not as important for low-frequency stimuli as it is for high-frequency stimuli. However, signal detection theory assumes a rate code and can be applied in cases in which spike timing is not important. Our results show that an optimal detector receiving a single P-unit spike train with baseline negative ISI correla- tions can detect the presence of stimuli that would give rise to as few as two extra spikes over a counting time of $\sim 250$ EOD cycles, as suggested by Ratnam and Nelson (2000). For low-frequency stimuli, signal detection theory is appropriate because the change in mean firing rate (computed over an appropriate time window) without a concomitant change in firing rate variance can signal the presence or absence of the stimulus. Without a change in variance, there will be no change in entropy (because the entropy of a random variable does not depend on its mean) (Shannon, 1948) and thus almost no mutual information as per our measure. For zero-mean high-frequency stimuli, there may be almost no change in mean firing rate computed over a long time window; hence, signal detection theory is not appropriate. However, because baseline entropy is high (this is caused by the high variability at short counting times) and because ISI correlations reduce the noise entropy, the mutual information will be high. The ideas presented above are compatible with a recent analysis by Salinas and Sejnowksi (2000) in which neurons can be driven either by the mean excitatory level (mean firing rate as assessed by the PND in our case) or by fluctuations around this mean (as assessed by mutual information in our case). Hence, the high variability of P-afferent spike trains observed for short counting times gives a high mutual information when looking at high-frequency stimuli, whereas the low variability at longer counting times caused by negative ISI correlations at short lags improves signal detectability at low frequencies.

\section{Conclusion and outlook}

We have shown that negative ISI correlations that are seen experimentally can improve the ability of a neuron to code both slow and fast time-varying stimuli. The P-units we have studied are known to converge onto basilar pyramidal cells of the electrosensory lateral line lobe (ELL) (Bastian, 1981). Population averaging is thus expected and might explain the extreme behavioral sensitivity to AMs, down to $0.1 \%$ of baseline EOD (Knudsen, 1974; Nelson and MacIver, 1999). Also, as mentioned before, the position of the minimum of the Fano factor is highly variable (40-1000 EOD cycles). Moreover, it is possible that different P-units (probability of firing per EOD cycle ranges from 0.1 to 0.5) (Nelson et al., 1997) will have different cumulative relative refractoriness decay time constants. It will thus be very interesting to study ELL decoding of slowly versus rapidly time-varying input processed by this heterogeneous P-unit population.

\section{APPENDIX A}

In this appendix, we give a list of all the symbols and acronyms used in this paper. When a symbol is constant throughout the paper (e.g., model parameter), its value is given. Units are only given for symbols.

\begin{tabular}{lll}
$\begin{array}{l}\text { Symbol/ } \\
\text { acronym }\end{array}$ & Description & Value/units \\
\hline$\beta$ & Constant & 1 (spikes/EOD cycle) ${ }^{-1}$ \\
$\Delta \tau$ & Bin width & 1 EOD cycle \\
$\Delta \theta$ & Constant by which threshold & 0.05 \\
& $\quad$ is incremented & \\
$\gamma$ & Constant & $0.3266(\mathrm{mV})^{-1}$ \\
$\xi_{1}$ & Uncorrelated Gaussian & Dimensionless \\
& $\quad$ random variable & \\
$\xi_{2}$ & Uncorrelated Gaussian & Dimensionless \\
& random variable & \\
\hline
\end{tabular}


Symbol/ acronym

$\lambda_{1} \quad$ Ornstein-Uhlenbeck process

$\lambda_{2} \quad$ Ornstein-Uhlenbeck process

$\mu \quad$ Mean of the spike count

$\rho_{\mathrm{j}} \quad$ Serial correlation coefficient at lag $\mathrm{j}$

$\sigma \quad$ SD of the spike count

$\sigma_{\text {stim }} \quad$ Stimulus contrast

$\theta$

$\theta_{0}$

$\tau_{1}$

$\tau_{2}$

$\tau_{a}$

$\tau_{b}$

$\tau_{v}$

$\tau_{\theta}$

$\Theta$

$A(t)$

$A_{0}$

AM

$c$

$C_{1}$

$C_{2}$

$\mathrm{CV}$

$d$

$d_{\text {crit }}$

$D_{1}$

$\mathrm{D}_{2}$

$d t \quad$ Integration time step

EOD Electric organ discharge

F

$f$

$f_{c}$

$f_{\mathrm{EOD}}$

$G_{a}$

$G_{b}$

$G_{c}$

H

I

$I_{c}$

\section{$I_{i}$}

$I_{\text {syn }}$

$\langle I\rangle$

ISI

ISIH

L

LIF

LIFDT

Threshold for the LIFDT model

Equilibrium value of the threshold

Time constant of $\lambda_{1}$

Time constant of $\lambda_{2}$

Time constant in Equation 3

Time constant in Equation 4

Voltage decay time constant

Threshold decay time constant

Heaviside function

Amplitude modulation of the EOD

Baseline EOD amplitude

Amplitude modulation

(of the EOD)

Constant

Fitting constant

Fitting constant

Coefficient of variation

Discriminability

Discriminability threshold

Intensity of $\lambda_{1}$

Intensity of $\lambda_{2}$

\section{Fano factor}

Frequency

Stimulus cutoff frequency

EOD frequency

Gain term in Equation 3

Gain term in Equation 4

Gain term in Equation 5

Entropy or entropy rate

Mutual information or mutual information rate ted during the correlation

time of the stimulus

$$
\text { ith ISI }
$$

Synaptic current in LIFDT model

Mean of the ISI distribution

$$
\text { Interspike interval }
$$

Interspike interval histogram

Word length

Leaky integrate-and-fire
Value/units

Dimensionless
Dimensionless
Spikes
Dimensionless
Spikes
$\mathrm{mV}$

Average information transmit-

Leaky integrate-and-fire with dynamic threshold

EOD cycles

\begin{tabular}{|c|c|c|}
\hline acronym & Description & Value/units \\
\hline$m$ & $\begin{array}{l}\text { Number of subprocesses used } \\
\text { in Nelson's model }\end{array}$ & 18 \\
\hline$p$ & $\begin{array}{l}\text { Probability of firing per } \\
\text { EOD cycle }\end{array}$ & Dimensionless \\
\hline$P_{D}$ & $\begin{array}{l}\text { Probability of correct } \\
\text { detection }\end{array}$ & Dimensionless \\
\hline$P_{\mathrm{FA}}$ & Probability of false alarm & Dimensionless \\
\hline PND & Pulse number distribution & \\
\hline$r$ & $\begin{array}{l}\text { Time-dependent firing rate in } \\
\text { Nelson's model }\end{array}$ & $\mathrm{Hz}$ \\
\hline$r_{\text {base }}$ & $\begin{array}{l}\text { Baseline firing rate in Nelson's } \\
\text { model }\end{array}$ & $200 \mathrm{~Hz}$ \\
\hline ROC & $\begin{array}{l}\text { Receiver operating } \\
\text { characteristic }\end{array}$ & \\
\hline$s$ & Time-varying stimulus & Dimensionless \\
\hline SCC & Serial correlation coefficient & \\
\hline SDF & Spectral density function & Dimensionless \\
\hline$T$ & Counting time window length & EOD cycles \\
\hline$T_{r}$ & $\begin{array}{l}\text { Absolute refractory period } \\
\text { duration in LIFDT }\end{array}$ & 1 EOD cycle \\
\hline$v$ & $\begin{array}{l}\text { Membrane voltage in the } \\
\text { LIFDT model }\end{array}$ & Dimensionless (see text) \\
\hline VAR & $\begin{array}{l}\text { Variance of the ISI } \\
\text { distribution }\end{array}$ & $(\text { EOD cycles })^{2}$ \\
\hline$X$ & Filtered stimulus & Spikes/EOD cycle \\
\hline$X_{a}$ & Filter variable in Equation 3 & Spikes/EOD cycle \\
\hline$X_{b}$ & Filter variable in Equation 4 & Spikes/EOD cycle \\
\hline
\end{tabular}

Dimensionless
Symbol/

\section{APPENDIX B}

In this appendix, we summarize the equations used for both models.

\section{Nelson model}

The AM $A(t)$ is filtered using the following set of equations:

$$
\begin{aligned}
& \dot{X}_{a}=-\frac{X_{a}}{\tau_{a}}+\frac{G_{a}}{\tau_{a}} A(t) \\
& \dot{X}_{b}=-\frac{X_{b}}{\tau_{b}}+\frac{G_{b}}{\tau_{b}} A(t) \\
& X(t)=-X_{a}-X_{b}+\left(G_{a}+G_{b}+G_{c}\right) A(t) .
\end{aligned}
$$

A baseline firing rate $r_{\text {base }}$ is added to $X(t)$ and $Z(t)=X(t)+r_{\text {base }}$ is rectified according to:

$$
r(t)= \begin{cases}0 & Z(t)<0 \\ f_{\mathrm{EOD}} & Z(t)>f_{\mathrm{EOD}} \\ Z(t) & \text { otherwise. }\end{cases}
$$

The probability of firing per EOD cycle is $p(t)=r(t) / f_{\mathrm{EOD}}$. Thus, at each maximum of the EOD, the P-unit has probability $p(t)$ of firing. If there is a firing, jitter is added to the spike time in the form of Gaussian white noise of zero mean and SD 0.04 EOD cycles. The ISIH can be made more regular by simulating $m$ copies of the subprocess and generating action potentials at the time of occurrence of the $m$ th event (Nelson et al., 1997). 


\section{LIFDT model}

In our model, the $\mathrm{AM} A(t)$ is filtered using the same set of equations as for the Nelson model:

$$
\begin{aligned}
& \dot{X}_{a}=-\frac{X_{a}}{\tau_{a}}+\frac{G_{a}}{\tau_{a}} A(t) \\
& \dot{X}_{b}=-\frac{X_{b}}{\tau_{b}}+\frac{G_{b}}{\tau_{b}} A(t) \\
& X(t)=-X_{a}-X_{b}+\left(G_{a}+G_{b}+G_{c}\right) A(t) .
\end{aligned}
$$

The total nondimensionalised synaptic current arriving at the spike initiation zone is given by:

$$
\begin{aligned}
& I_{\mathrm{syn}}=\left(\beta X(t)+\gamma A_{0}\right) \Theta\left(\beta X(t)+\gamma A_{0}\right) \sin \left(2 \pi f_{\mathrm{EOD}} t\right) \times \\
& \Theta\left(\sin \left(2 \pi f_{\mathrm{EOD}} t\right)\right)\left(1+\lambda_{1}\right)+\lambda_{2},
\end{aligned}
$$

where $\beta$ and $\gamma$ are constants to ensure units are matched, $A_{0}$ is the baseline EOD amplitude, $f_{\mathrm{EOD}}$ is the EOD frequency, and $\Theta$ is the Heaviside function used for rectification $[\Theta(x)=1$ if $x \geq 0$ and $\Theta(x)=0$ if $x<0]$. The noise sources $\lambda_{1}$ and $\lambda_{2}$ are Ornstein-Uhlenbeck processes given by [see text for a brief introduction and Gardiner (1985) for further information]:

$$
\begin{aligned}
& \dot{\lambda}_{1}=-\frac{\lambda_{1}}{\tau_{1}}+\sqrt{D_{1}} \xi_{1} \\
& \dot{\lambda}_{2}=-\frac{\lambda_{2}}{\tau_{2}}+\sqrt{D_{2}} \xi_{2} .
\end{aligned}
$$

The noise sources $\lambda_{1}$ and $\lambda_{2}$ are shown in Figure $2 a$. For example, the synaptic current for baseline dynamics is just a rectified sine-wave (i.e., the negative part is set to zero) perturbed by noise. In the time window between action potentials and after the absolute refractory period, the voltage $v$ and the threshold $\theta$ are given by:

$$
\begin{aligned}
& \dot{v}=-\frac{v}{\tau_{v}}+\frac{I_{\text {syn }}}{\tau_{v}} \\
& \dot{\theta}=\left(\theta_{0}-\theta\right) / \tau_{\theta} .
\end{aligned}
$$

A spike is said to have occurred when $v=\theta$. Immediately afterward, $v$ is reset to zero, and $\theta$ is incremented by a constant $\Delta \theta$. The threshold is then maintained constant for the duration of the absolute refractory period $T_{r}$ before decaying exponentially until the next spike time. A stretch of simulation showing voltage and threshold is shown in Figure 2.

\section{APPENDIX C}

In this appendix, we give an intuitive explanation of why adding a slow noise to our model will give rise to positive ISI correlations.

We first recall that the Ornstein-Uhlenbeck process $\lambda_{2}$ varies on a time scale much greater than either the voltage or threshold variables in the LIFDT model because $\tau_{2}=50,000$ EOD cycles is much greater than an EOD cycle. Thus, we can treat $\lambda_{2}$ as a quasistatic variable with respect to voltage and threshold because it is almost constant when considering time scales much smaller than $\tau_{2}$. Because the mean ISI is five EOD cycles long, many ISIs (10,000 on average) will have occurred during a time window of length $\tau_{2}$. Let us imagine that $\lambda_{2}$ has some value; then $\lambda_{2}$ will have that value (approximately) for a long time. If the value is positive, then the synaptic current $I_{\text {syn }}$ is bigger than it would be if $\lambda_{2}=0$ and we thus expect a long sequence of ISIs of shorter duration (but not much shorter because $\lambda_{2}$ is weak) than on average (when $\lambda_{2}=0$ ). When $\lambda_{2}$ has a negative value, one can expect sequences of ISIs longer than average by the same argument. Thus, we will get long sequences of ISIs that are shorter than average and long sequences of ISIs that are longer than average. It is these long sequences that will lead to positive SCCs (the SCC at a given lag is positive only when two ISIs separated by this lag are both shorter or longer than average). Because those sequences can be very long, the ISI correlations extend to long lags. As mentioned in the text, a full mathematical explanation will be presented elsewhere.

\section{REFERENCES}

Barlow HB, Levick WR (1969a) Three factors limiting the reliable detection of light by the retinal ganglion cells of the cat. J Physiol (Lond) 200:1-24.

Barlow HB, Levick WR (1969b) Changes in the maintained discharge with adaptation level in the cat retina. J Physiol (Lond) 202:699-718.

Bastian J (1981) Electrolocation I. How the electroreceptors of Apteronotus albifrons code for moving objects and other electrical stimuli. J Comp Physiol [A] 144:465-479.

Bastian J, Schneiderjen S, Nguyenkim J (2001) Arginine vasotocin modulates a sexually dimorphic communication behavior in the weakly electric fish, Apteronotus leptorhynchus. J Exp Biol, in press.

Bennett MVL, Sandri C, Akert K (1989) Fine structure of the tuberous electroreceptor of the high-frequency electric fish, Sternachus albifrons (gymnotiformes). J Neurocytol 18:265-283.

Berry II MJ, Meister M (1998) Refractoriness and neural precision. J Neurosci 18:2200-2211.

Bialek W, Rieke F, de Ruyter van Steveninck R, Warland D (1991) Reading a neural code. Science 252:1854-1857.

Borst A, Theunissen F (1999) Information theory and neural coding. Nat Neurosci 2:947-957.

Burčas GI, Albright TD (1999) Ganging sensory representation in the brain. Trends Neurosci 22:303-309.

Chacron MJ, Longtin A, St-Hilaire M, Maler L (2000) Suprathreshold stochastic firing dynamics with memory in P-type electroreceptors. Phys Rev Lett 85:1576-1579.

Chacron MJ, Longtin A, Maler L (2001) Simple models of bursting and non-bursting P-type electroreceptors. Neurocomputing, in press.

Chialvo DR, Longtin A, Muller-Gerking J (1997) Stochastic resonance in neuronal ensembles. Phys Rev E 55:1798-1808.

Cover T, Thomas J (1991) Elements of information theory. New York: Wiley.

Cox DR, Lewis PAW (1966) The statistical analysis of series of events. London: Methuen.

Dulka JG, Maler L, Ellis W (1995) Androgen-induced changes in electrocummunicatory behavior are correlated with changes in substance P-like immunoreactivity in the brain of the electric fish Apteronotus leptorhynchus. J Neurosci 15:1879-1890.

Fano U (1947) Ionization yield of radiations. II. The fluctuations of the number of ions. Physiol Rev 72:26-29.

French AS, Holden AV, Stein RB (1972) The estimation of the frequency response function of a mechanoreceptor. Kybernetik 11:15-23.

Gabbiani F (1996) Coding of time varying signals in spike trains of linear and half-wave rectifying neurons. Network 7:61-85.

Gabbiani F, Koch C (1996) Coding of time-varying signals in spike trains of integrate-and-fire neurons with random threshold. Neural Comput $8: 44-66$.

Gabbiani F, Koch C (1998) Principles of spike train analysis. In: Methods in neuronal modeling: from ions to networks (Koch C, Segev I, eds), pp 313-360. Cambridge, MA: MIT.

Gabbiani F, Metzner W (1999) Encoding and processing of sensory information in neuronal spike trains. J Exp Biol 202:1267:1279.

Gardiner CW (1985) Handbook of stochastic methods. Berlin: Springer.

Geisler CD, Goldberg JM (1966) A stochastic model of the repetitive activity of neurons. Biophys J 6:53-69.

Gestri G, Masterbroek HAK, Zaagman WH (1980) Stochastic constancy, variability and adaptation of spike generation: performance of a giant neuron in the visual system of the fly. Biol Cybern 38:31-40.

Green D, Swets J (1966) Signal detection theory and psychophysics. New York: Wiley.

Hausser M, Roth A (1997) Dendritic and somatic glutamate receptor channels in rat cerebellar Purkinje cells. J Physiol (Lond) 501:77-95.

Hopkins CD (1976) Stimulus filtering and electroreception: tuberous electroreceptors in three species of gymnotid fish. J Comp Physiol [A] 111:171-207.

Knudsen EI (1974) Behavioral thresholds to electric signals in high frequency electric fish. J Comp Physiol 91:333-353.

Koch C (1999) Biophysics of computation. New York: Oxford UP.

Kreiman G, Krahe R, Metzner W, Koch C, Gabbiani F (2000) Robust- 
ness and variability of neuronal coding by amplitude-sensitive afferents in the weakly electric fish Eigenmannia. J Neurophysiol 84:189-224.

Longtin A, Racicot DM (1997) Spike train patterning and forecastability. Biosystems 40:111-118.

Lowen SB, Teich MC (1992) Auditory nerve action potentials form a nonrenewal point process over short as well as long time scales. J Acoust Soc Am 92:803-806.

Lowen SB, Teich MC (1996) The periodogram and Allan variance reveal fractal exponents greater than unity in auditory-nerve spike trains. J Acoust Soc Am 99:3585-3591.

Lowen SB, Cash SS, Poo M, Teich MC (1997) Quantal neurotransmitter secretion rate exhibits fractal behavior. J Neurosci 17:5666-5677.

Manwani A, Koch C (1999) Detecting and estimating signals in noisy cable structures. I: Neuronal noise sources. Neural Comput 11:1797-1829.

Metzner W, Heiligenberg W (1991) The coding of signals in the electric communication of the gymnotiform fish Eigenmannia: from electroreceptors to neurons in the torus semicircularis of the midbrain. J Comp Physiol [A] 169:135-150.

Middlebrooks JC, Clock AE, Xu L, Green DM (1994) A panoramic code for sound location by cortical neurons. Science 264:842-844.

Moortgat KT, Keller CH, Bullock TH, Sejnowski TJ (1998) Submicrosecond pacemaker precision is behaviorally modulated: the gymnotiform electromotor pathway. Proc Natl Acad Sci USA 95:4684-4689.

Nachimas J (1972) Signal detection theory and its application to problems in vision. In: Visual Psychophysics (Jameson D, Hurvitch LM, eds) Ed 7 Vol 4 pp 56-77. Berlin: Springer.

Nelson ME, MacIver MA (1999) Prey capture in the weakly electric fish Apteronotus albifrons: sensory acquisition strategies and electrosensory consequences. J Exp Biol 202:1195-1203.

Nelson ME, Xu Z, Payne JR (1997) Characterization and modeling of P-type electrosensory afferent responses to amplitude modulations in a wave-type electric fish. J Comp Physiol [A] 181:532-544.

Ratnam R, Nelson ME (2000) Non-renewal statistics of electrosensory afferent spike trains: implications for the detection of weak sensory signals. J Neurosci 20:6672-6683.

Reinagel P, Reid RC (2000) Temporal coding of visual information in the thalamus. J Neurosci 20:5392-5400.

Rieke F, Warland D, de Ruyter van Steveninck R, Bialek W (1997) Spikes: exploring the neural code. Cambridge, MA: MIT.

Salinas E, Sejnowski TJ (2000) Impact of correlated synaptic input on output firing rate and variability in simple neuronal models. J Neurosci 20:6193-6209.

Scheich H, Bullock TH, Hamstra RH (1973) Coding properties of two classes of afferent nerve fibers: high frequency electroreceptors in the electric fish, Eigenmannia. J Neurophysiol 36:39-60.
Shannon CE (1948) A mathematical theory of communication. Bell Syst Tech J 27:379-423, 623-656.

Shofner WP, Dye RH (1989) Statistical and receiver operating characteristic analysis of empirical spike count distributions: quantifyiing the ability of cochlear nucleus units to original intensity changes. J Acoust Soc Am 86:2172-2184.

Snippe HP, Koenderink JJ (1992) Discrimination thresholds for channel-coded systems. Biol Cybern 66:543-551.

Softly WR, Koch C (1993) The highly irregular firing of cortical cells is inconsistent with temporal integration of EPSPs. J Neurosci 13:334350 .

Stein RB (1965) A theoretical analysis of neuronal variability. Biophys J 5:173-194.

Strong SP, Koberle R, de Ruyter van Steveninck RR, Bialek W (1998) Entropy and information in neural spike trains. Phys Rev Lett 80:197-200.

Teich MC (1992) Fractal neuronal firing patterns. In: Single neuron computation (McKenna T, Davis J, Zornetzer SF, eds), pp 589-622. San Diego: Academic.

Teich MC, Khanna SM (1985) Pulse-number distributions for the neural spike train in the cat's auditory nerve. J Acoust Soc Am 77:1110-1128.

Teich MC, Turcott RG, Siegel RM (1996) Temporal correlations in cat striate-cortex neural spike trains. IEEE Eng Med Biol Mag 15:79-87.

Teich MC, Heneghan C, Lowen SB, Ozaki T, Kaplan E (1997) Fractal character of the neural spike train in the visual system of the cat. J Opt Soc Am A 14:529-546.

Turcott RG, Teich MC (1996) Fractal character of the electrocardiogram: distinguishing heart-failure and normal patients. Ann Biomed Eng 24:269-293.

von Gernsdoff H, Schneggenburger R, Weis S, Neher E (1997) Presynaptic depression at a calyx synapse: the small contribution of metabotropic glutamate receptors. J Neurosci 17:8137-8146.

Wang LY, Gan L, Forsythe ID, Kaczmarek LK (1998) Contribution of the Kv3.1 potassium channel to high-frequency firing in mouse auditory neurones. J Physiol (Lond) 509:183-194.

Wessel R, Koch C, Gabbiani F (1996) Coding of time varying electric field amplitude modulations in a wave-type electric fish. J Neurophysiol 75:2280-2293.

Xu Z, Payne JR, Nelson ME (1996) Logarithmic time course of sensory adaptation in electrosensory afferent nerve fibers in a weakly electric fish. J Neurophysiol 96:2020-2032.

Zakon HH (1986) The electroreceptive periphery. In: Electroreception (Bullock TH, Heiligenberg W, eds), pp 103-156. New York: Wiley.

Zupanc GKH, Maler L (1993) Evoked chirping in the weakly electric fish Apteronotus leptorhynchus: a quantitative biophysical analysis. Can J Zool 71:2301-2310. 